\title{
Carbon Nitride Transforms into a High Lithium- Storage Capacity Nitrogen-Rich Carbon
}

Joshua P. Pender, ${ }^{\zeta}$ Joseph V. Guerrera, ${ }^{\zeta}$ Bryan R. Wygant,${ }^{\zeta}$ Jason A. Weeks, ${ }^{\zeta}$ Ryan A. Ciufo, ${ }^{\zeta}$ James N. Burrow, ${ }^{\delta}$ Mitchell F. Walk, ${ }^{\delta}$ Mohammad Z. Rahman, ${ }^{\delta}$ Adam Heller, ${ }^{\delta}$ and C. Buddie Mullins* ${ }^{*} \zeta, \delta$

¿Department of Chemistry and ${ }^{\delta} \mathrm{J}$ ohn J. McKetta Department of Chemical Engineering, The University of Texas at Austin 78712, USA.

*Corresponding author: Prof. C. Buddie Mullins

Email: mullins@che.utexas.edu 
S-3. Figure S1: SEM characterization of bulk $\mathrm{C}_{3} \mathrm{~N}_{4}$ precursor

S-4. Figure S2: Additional SEM characterization of $\mathrm{C}_{3} \mathrm{~N}_{4}-\mathrm{Cu}$ samples

S-5. Figure S3: Additional SEM characterization of $\mathrm{C}_{3} \mathrm{~N}_{4}-\mathrm{Fe}$ samples

S-6. Figure S4 and Table S1: Raman fitting of D-band and G-band for $\mathrm{C}_{3} \mathrm{~N}_{4}-\mathrm{Cu}$ carbons

S-7. Figure S5 and Table S2: Raman fitting of D-band and G-band for $\mathrm{C}_{3} \mathrm{~N}_{4}-\mathrm{Fe}$ carbons

S-8. Figure S6. High-resolution C 1s and N 1s XPS spectra of bulk $\mathrm{C}_{3} \mathrm{~N}_{4}$

S-9. Figure S7: High resolution $\mathrm{C} 1 \mathrm{~s}$ and $\mathrm{N} 1 \mathrm{~s}$ XPS spectra of $\mathrm{C}_{3} \mathrm{~N}_{4}-\mathrm{Cu}$ carbons

S-10. Figure S8: High-resolution $C 1$ s and N 1s XPS spectra of $\mathrm{C}_{3} \mathrm{~N}_{4}-\mathrm{Fe}$

S-11. Figure S9: Residual Gas Analysis (RGA) of $\mathrm{C}_{3} \mathrm{~N}_{4}-\mathrm{Cu}$ and $\mathrm{C}_{3} \mathrm{~N}_{4}-\mathrm{Fe}$

S-12. Figure S10: Thermogravimetric Analysis (TGA) curves of $\mathrm{C}_{3} \mathrm{~N}_{4}-\mathrm{Cu} 750$ and $\mathrm{C}_{3} \mathrm{~N}_{4}-\mathrm{Fe} 750$

S-13. Figure S11: Cyclic voltammetry (CV) analysis of bulk $\mathrm{C}_{3} \mathrm{~N}_{4}$

S-14. Figure S12: Galvanostatic charge-discharge analysis of bulk $\mathrm{C}_{3} \mathrm{~N}_{4}$

S-15. Figure S13: Coulombic efficiency vs. cycle for $0.1-20 \mathrm{~A} \mathrm{~g}^{-1}$ (see Figure 7C) and chargedischarge profiles for $\mathrm{C}_{3} \mathrm{~N}_{4}-\mathrm{Cu} 700-800$ at $0.1,0.5,1.0,5.0$, and $20.0 \mathrm{~A} \mathrm{~g}^{-1}$

S-16. Figure S14: Coulombic efficiency vs. cycle for $0.1-20 \mathrm{~A} \mathrm{~g}^{-1}$ (see Figure 7D) and chargedischarge profiles for $\mathrm{C}_{3} \mathrm{~N}_{4}-\mathrm{Fe} 00-800$ at $0.1,0.5,1.0,5.0$, and $20.0 \mathrm{~A} \mathrm{~g}^{-1}$

S-17. Figure S15: $d Q d^{-1}$ vs. V plots for $\mathrm{C}_{3} \mathrm{~N}_{4}-\mathrm{Cu} 700-800$ and $\mathrm{C}_{3} \mathrm{~N}_{4}-\mathrm{Fe} 700-800$ at $0.1,0.2,0.5$, and $1.0 \mathrm{~A} \mathrm{~g}^{-1}$

S-18. Equation S1 and S2: Cyclic voltammetry analysis

S-19. Figure S16: Variable scan-rate cyclic voltammetry and b-value analysis for $\mathrm{C}_{3} \mathrm{~N}_{4}-\mathrm{Cu} 700$ 800

S-20. Figure S17: Variable scan-rate cyclic voltammetry and b-value analysis for $\mathrm{C}_{3} \mathrm{~N}_{4}-\mathrm{Fe} 700$ 800

S-21. Figure S18: Charge-discharge curves and coulombic efficiency plots for $\mathrm{C}_{3} \mathrm{~N}_{4}-\mathrm{Cu} 700-800$ carbons over 200 cycles at $0.1 \mathrm{~A} \mathrm{~g} \mathrm{~g}^{-1}\left(0.2 \mathrm{~mA} \mathrm{~cm}^{-2}\right)$

S-22. Figure S19: Charge-discharge curves and coulombic efficiency plots for $\mathrm{C}_{3} \mathrm{~N}_{4}-\mathrm{Fe} 700-800$ carbons over 200 cycles at $0.1 \mathrm{~A} \mathrm{~g}^{-1}\left(0.2 \mathrm{~mA} \mathrm{~cm}^{-2}\right)$

S-23. Figure S20: Charge-discharge curves and coulombic efficiency plots for $\mathrm{C}_{3} \mathrm{~N}_{4}-\mathrm{Cu} 700-800$ carbons over 2000 cycles at $2.0 \mathrm{~A} \mathrm{~g}^{-1}\left(4.0 \mathrm{~mA} \mathrm{~cm}^{-2}\right)$

S-24. Figure S21: Charge-discharge curves and coulombic efficiency plots for $\mathrm{C}_{3} \mathrm{~N}_{4}-\mathrm{Fe} 700-800$ carbons over 2000 cycles at $2.0 \mathrm{~A} \mathrm{~g}^{-1}\left(4.0 \mathrm{~mA} \mathrm{~cm}^{-2}\right)$

S-25. Figure S22: Specific Capacity vs. Cycle between 0.1 to $20 \mathrm{~A} \mathrm{~g}^{-1}$ for $\mathrm{C}_{3} \mathrm{~N}_{4}-\mathrm{Cu} 750$ at different mass loading

S-26. Table S3. Parameters from previous works used to construct Figure 10b.

S-27. Figure S23: Electrochemical Impedance Spectroscopy (EIS) Analysis of bulk $\mathrm{C}_{3} \mathrm{~N}_{4}, \mathrm{C}_{3} \mathrm{~N}_{4}$ $\mathrm{Cu}$, and $\mathrm{C}_{3} \mathrm{~N}_{4}-\mathrm{Fe}$ carbons

S-28. References 


\section{Bulk $\mathrm{C}_{3} \mathrm{~N}_{4}$}
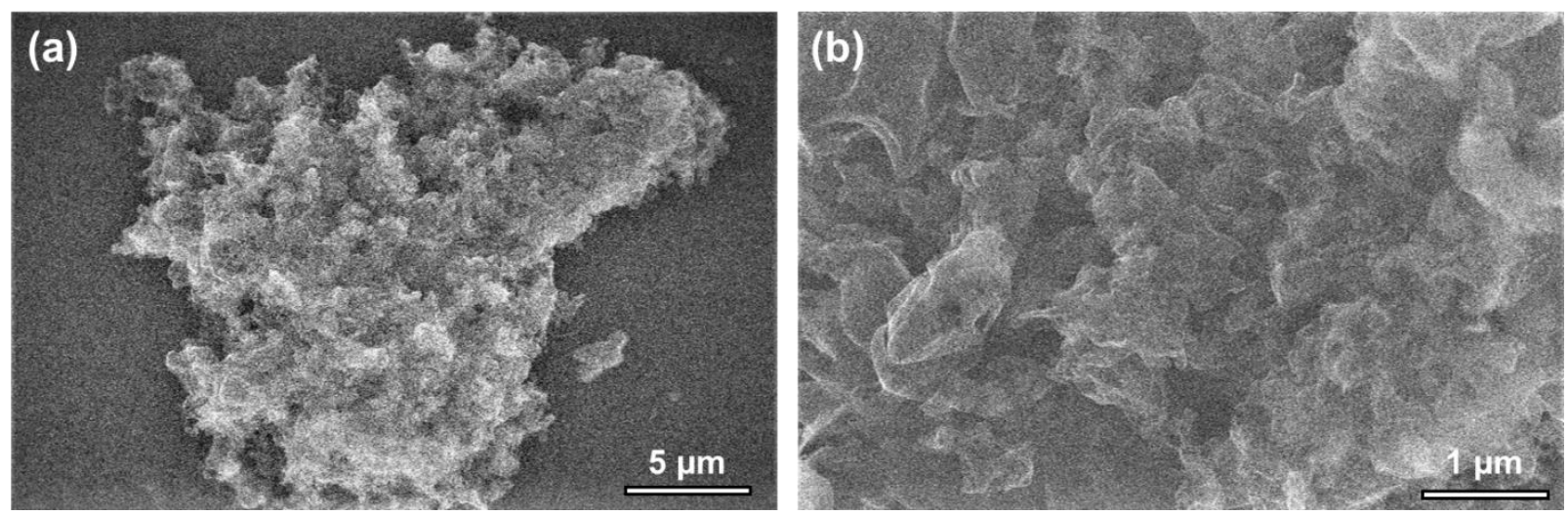

Figure S1. Representative SEM images of bulk $\mathrm{C}_{3} \mathrm{~N}_{4}$ synthesized from urea at $550^{\circ} \mathrm{C}$ for 4 hours with a $2.3^{\circ} \mathrm{C} \mathrm{min}{ }^{-1}$ ramp rate. (a) Image showing average $C_{3} \mathrm{~N}_{4}$ particle size of $\sim 15-20 \mu \mathrm{m}$ and (b) highmagnification showing the surface of a $\mathrm{C}_{3} \mathrm{~N}_{4}$ particle. 

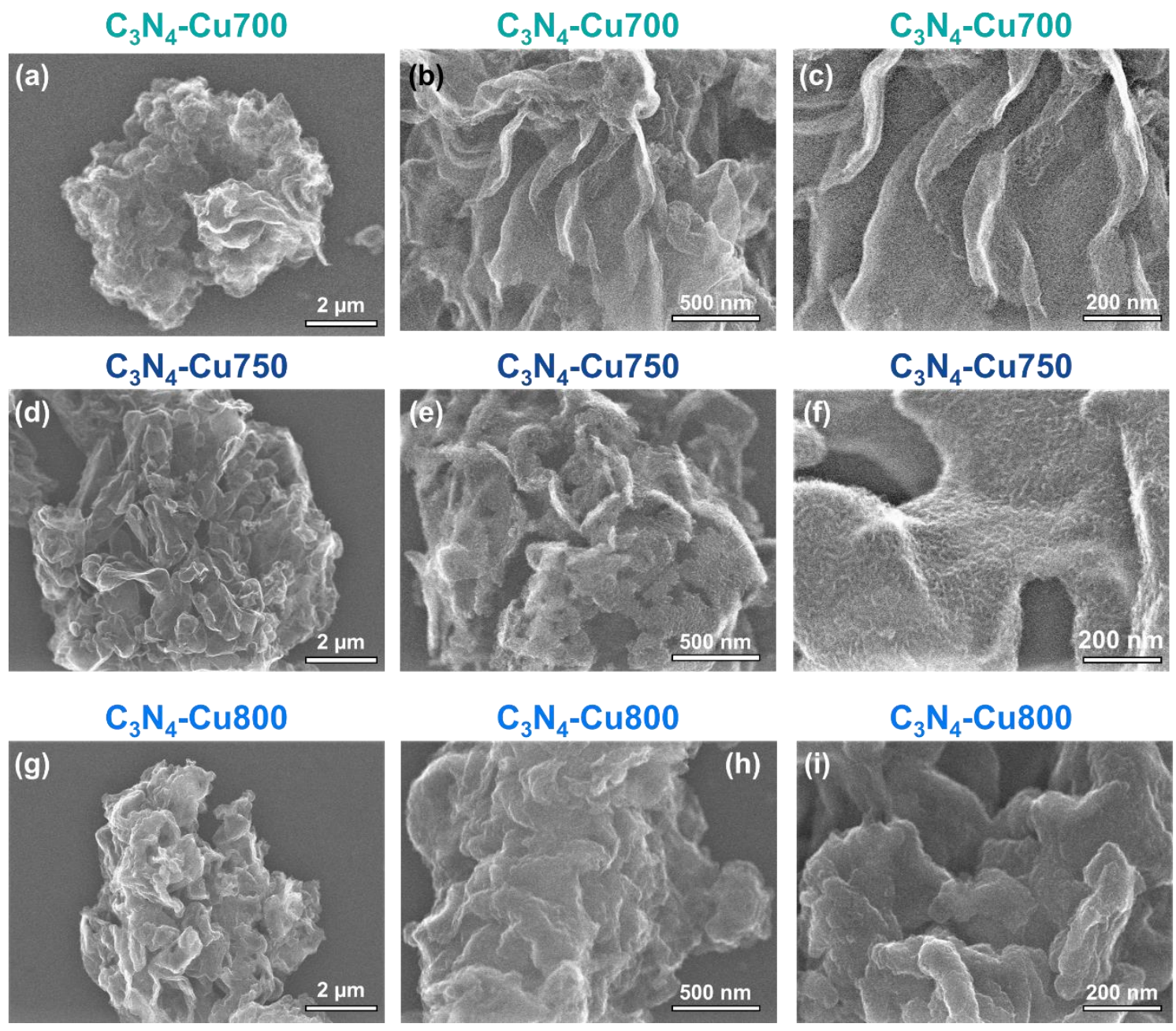

Figure S2. SEM images at different magnification for (a-c, top row) $\mathrm{C}_{3} \mathrm{~N}_{4}-\mathrm{Cu} 700$, (d-f, middle row) $\mathrm{C}_{3} \mathrm{~N}_{4}-\mathrm{Cu} 750$, and (g-i, bottom row) $\mathrm{C}_{3} \mathrm{~N}_{4}-\mathrm{Cu} 800$. 

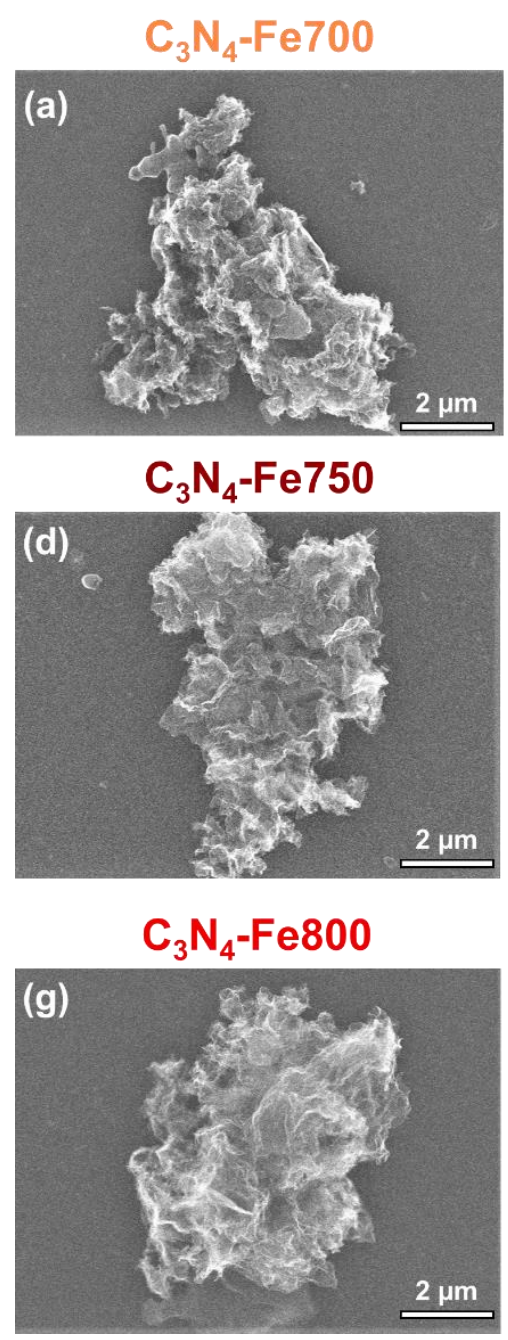
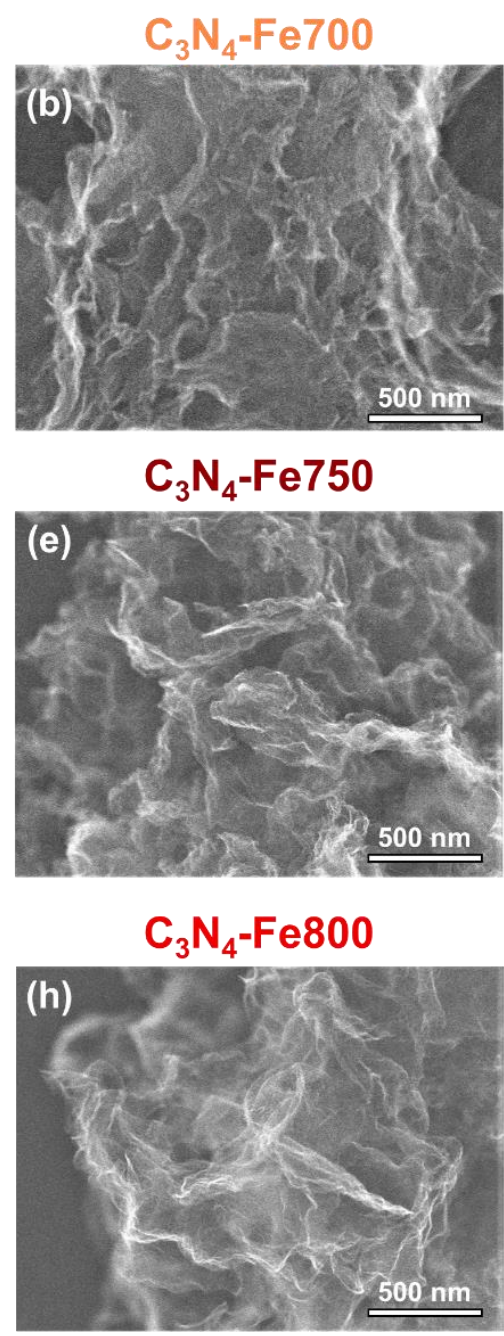

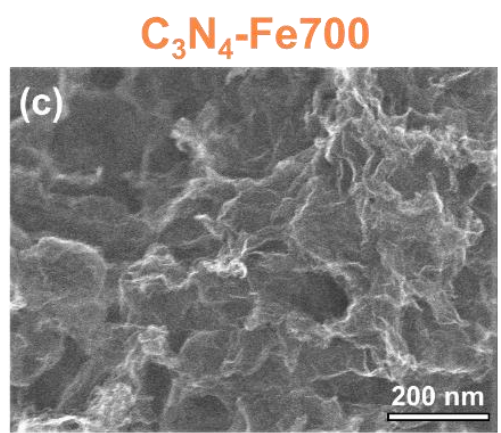

$\mathrm{C}_{3} \mathrm{~N}_{4}-\mathrm{Fe} 750$

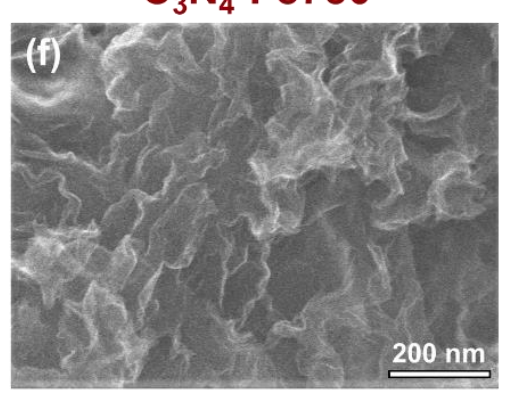

$\mathrm{C}_{3} \mathrm{~N}_{4}-\mathrm{Fe} 800$

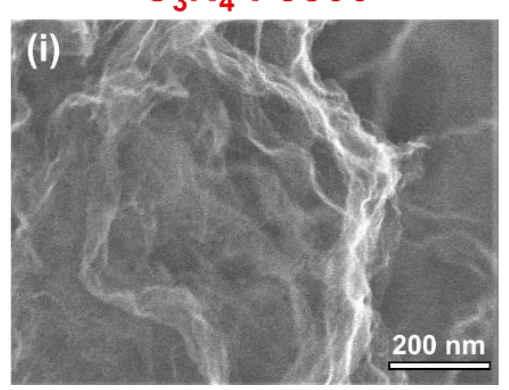

Figure S3. SEM images at different magnification for (a-c, top row) $\mathrm{C}_{3} \mathrm{~N}_{4}-\mathrm{Fe} 700$, (d-f, middle row) $\mathrm{C}_{3} \mathrm{~N}_{4}-\mathrm{Fe} 750$, and (g-i, bottom row) $\mathrm{C}_{3} \mathrm{~N}_{4}-\mathrm{Fe} 800$. 


\section{Raman fitting of D-band and G-band for $\mathrm{C}_{3} \mathrm{~N}_{4}-\mathrm{Cu}$ carbons}


Figure S4. Voigt fits of D-band and G-band region from Raman spectroscopy for (a) $\mathrm{C}_{3} \mathrm{~N}_{4}-\mathrm{Cu} 700$, (b) $\mathrm{C}_{3} \mathrm{~N}_{4}-\mathrm{Cu} 750$, and (c) $\mathrm{C}_{3} \mathrm{~N}_{4}-\mathrm{Cu} 800$.

Table S1. Summary of Raman spectroscopy values obtained from Voigt Fits for $\mathrm{C}_{3} \mathrm{~N}_{4}-\mathrm{Cu} 700 \mathrm{C}_{3} \mathrm{~N}_{4-}$ $\mathrm{Cu} 750$, and $\mathrm{C}_{3} \mathrm{~N}_{4}-\mathrm{Cu} 800$.

\begin{tabular}{|c|c|c|c|c|c|}
\hline Material & $\begin{array}{c}\text { D-band } \\
\text { position } \\
\left(\mathrm{cm}^{-1}\right)\end{array}$ & $\begin{array}{c}\text { D-band } \\
\text { FWHM } \\
\left(\mathrm{cm}^{-1}\right)\end{array}$ & $\begin{array}{c}\text { G-band } \\
\text { position } \\
\left(\mathrm{cm}^{-1}\right)\end{array}$ & $\begin{array}{c}\text { G-band } \\
\text { FWHM } \\
\left(\mathrm{cm}^{-1}\right)\end{array}$ & $\mathrm{I}_{\mathrm{D}} / \mathrm{I}_{\mathrm{G}}$ \\
\hline $\mathrm{C}_{3} \mathrm{~N}_{4}-\mathrm{Cu} 700$ & 1375.8 & 163.0 & 1584.3 & 120.7 & 1.16 \\
\hline $\mathrm{C}_{3} \mathrm{~N}_{4}-\mathrm{Cu} 750$ & 1381.7 & 144.1 & 1587.1 & 92.5 & 1.19 \\
\hline $\mathrm{C}_{3} \mathrm{~N}_{4}-\mathrm{Cu} 800$ & 1387.9 & 148.9 & 1605.4 & 87.4 & 1.13 \\
\hline
\end{tabular}



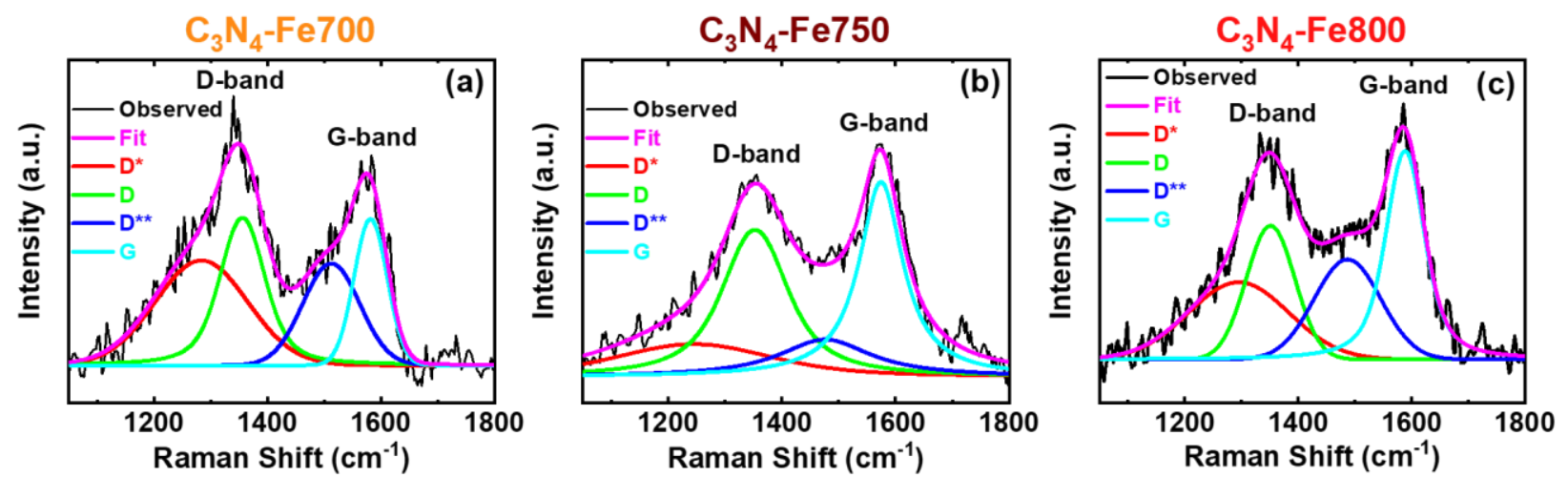

Figure S5. Voigt fits of D-band and G-band region from Raman spectroscopy for (a) $\mathrm{C}_{3} \mathrm{~N}_{4}-\mathrm{Fe} 700$, (b) $\mathrm{C}_{3} \mathrm{~N}_{4}-\mathrm{Fe} 750$, and (c) $\mathrm{C}_{3} \mathrm{~N}_{4}-\mathrm{Fe} 800$.

Table S2. Summary of Raman spectroscopy values obtained from Voigt Fits for $\mathrm{C}_{3} \mathrm{~N}_{4}-\mathrm{Fe} 700 \mathrm{C}_{3} \mathrm{~N}_{4}$ $\mathrm{Fe} 750$, and $\mathrm{C}_{3} \mathrm{~N}_{4}-\mathrm{Fe} 800$.

\begin{tabular}{|c|c|c|c|c|c|}
\hline Material & $\begin{array}{c}\text { D-band } \\
\text { position } \\
\left(\mathrm{cm}^{-1}\right)\end{array}$ & $\begin{array}{c}\text { D-band } \\
\text { FWHM } \\
\left(\mathrm{cm}^{-1}\right)\end{array}$ & $\begin{array}{c}\text { G-band } \\
\text { position } \\
\left(\mathrm{cm}^{-1}\right)\end{array}$ & $\begin{array}{c}\text { G-band } \\
\text { FWHM } \\
\left(\mathrm{cm}^{-1}\right)\end{array}$ & $\mathrm{I}_{\mathrm{D}} / \mathrm{I}_{\mathrm{G}}$ \\
\hline $\mathrm{C}_{3} \mathrm{~N}_{4}-\mathrm{Fe} 700$ & 1355.8 & 192.0 & 1581.5 & 94.1 & 1.01 \\
\hline $\mathrm{C}_{3} \mathrm{~N}_{4}-\mathrm{Fe} 750$ & 1352.8 & 137.8 & 1584.4 & 89.1 & 0.74 \\
\hline $\mathrm{C}_{3} \mathrm{~N}_{4}-\mathrm{Fe} 800$ & 1351.1 & 100.9 & 1588.4 & 81.2 & 0.70 \\
\hline
\end{tabular}


XPS Fitting Parameters: High-resolution XPS scans were analyzed using CasaXPS fitting software. The high-resolution $\mathrm{C} 1 \mathrm{~s}$ and $\mathrm{N}$ 1s spectra for bulk $\mathrm{C}_{3} \mathrm{~N}_{4}$ (Figure $\mathrm{S} 6$ ), $\mathrm{C}_{3} \mathrm{~N}_{4}-\mathrm{Cu}$ (Figure $\mathrm{S} 7$ ), and $\mathrm{C}_{3} \mathrm{~N}_{4}-\mathrm{Cu}$ (Figure S8) were deconvoluted assuming a Shirley background correction, and peaks were fit to a 30/70 Gaussian-Lorentzian fitting function.

\section{XPS characterization of bulk $\mathrm{C}_{3} \mathrm{~N}_{4}$}
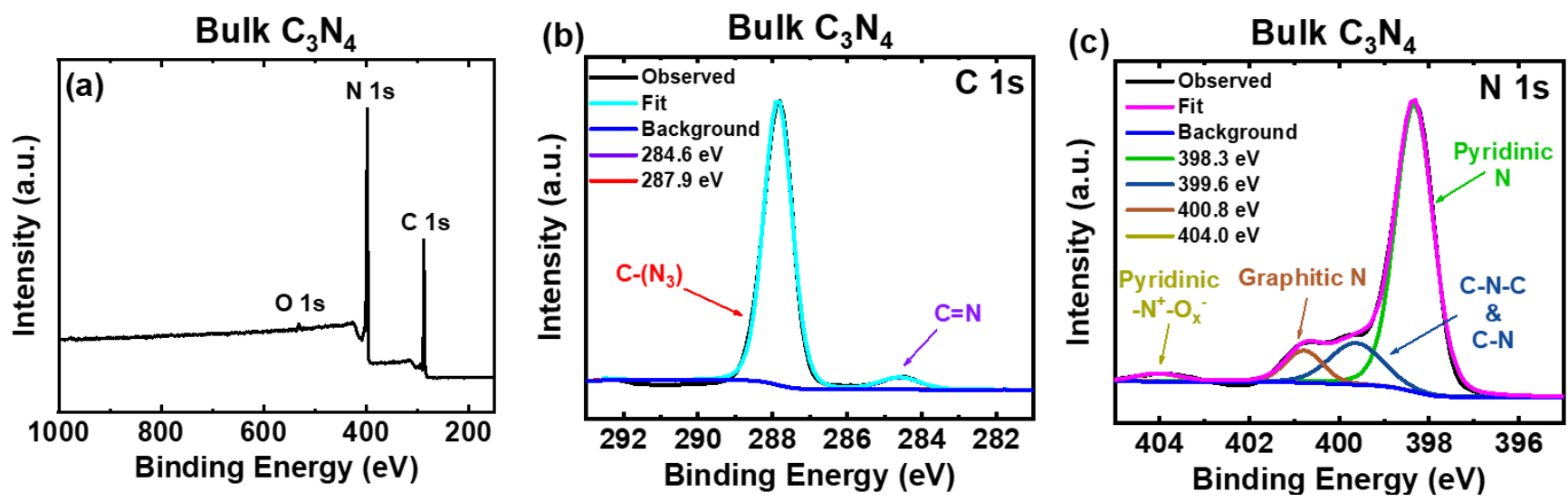

Figure S6. XPS characterization of bulk $\mathrm{C}_{3} \mathrm{~N}_{4}$. (a) XPS Survey scan, (b) high-resolution $\mathrm{C}$ 1s spectrum, and (c) high-resolution $\mathrm{N}$ 1s spectrum. 


\section{High resolution $\mathrm{C}$ 1s and $\mathrm{N}$ 1s (XPS) characterization of $\mathrm{C}_{3} \mathrm{~N}_{4}-\mathrm{Cu}$ carbons}
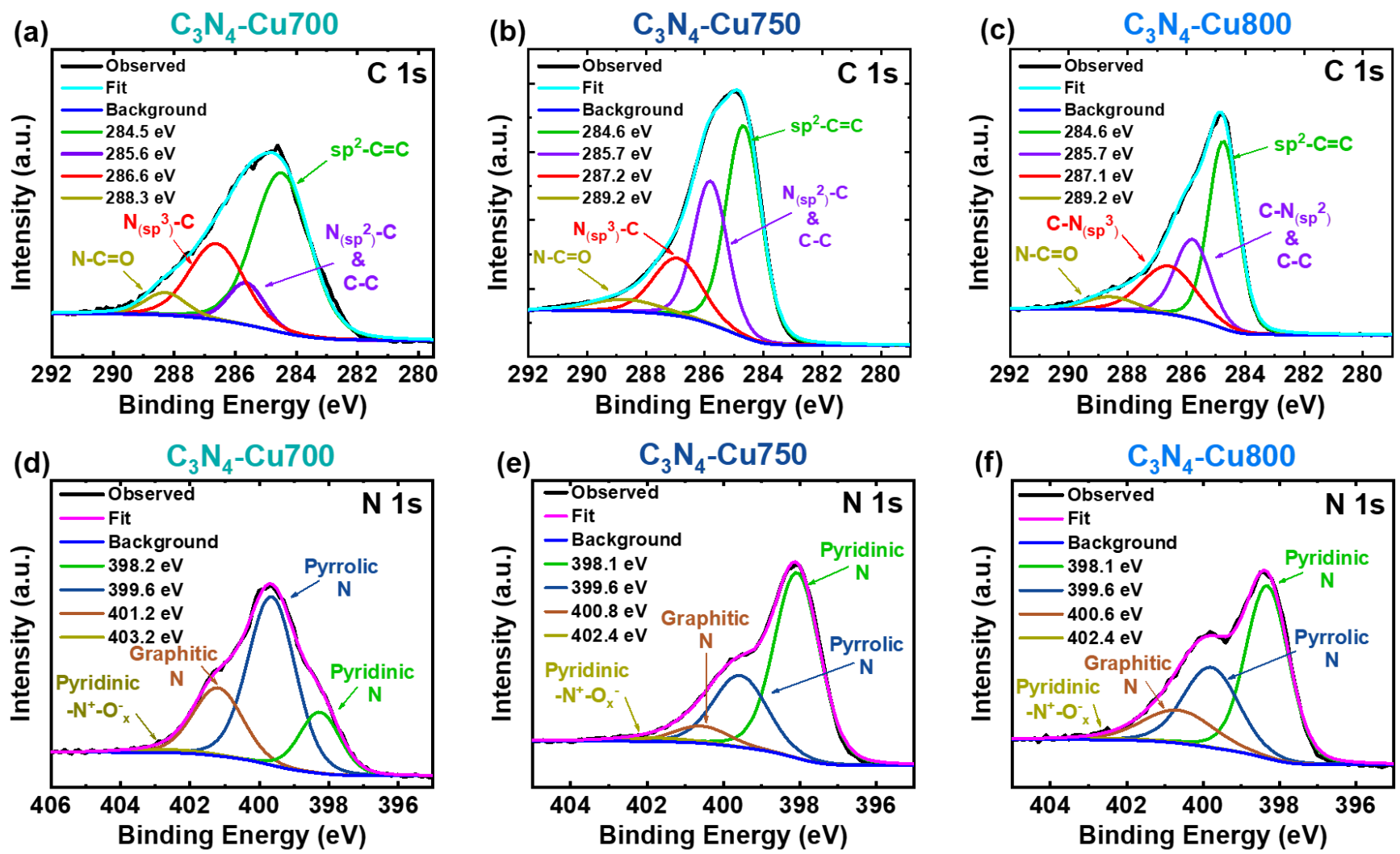

Figure S7. High resolution XPS C 1s (a-c, top row) and N 1s (d-f, bottom row) spectra of $\mathrm{C}_{3} \mathrm{~N}_{4}-\mathrm{Cu} 700$ (a, d), $\mathrm{C}_{3} \mathrm{~N}_{4}-\mathrm{Cu} 750(\mathrm{~b}, \mathrm{e})$, and $\mathrm{C}_{3} \mathrm{~N}_{4}-\mathrm{Cu} 800$ (c, f). 
High resolution $\mathrm{C}$ 1s and $\mathrm{N}$ 1s (XPS) characterization of $\mathrm{C}_{3} \mathrm{~N}_{4}-\mathrm{Fe}$ carbons
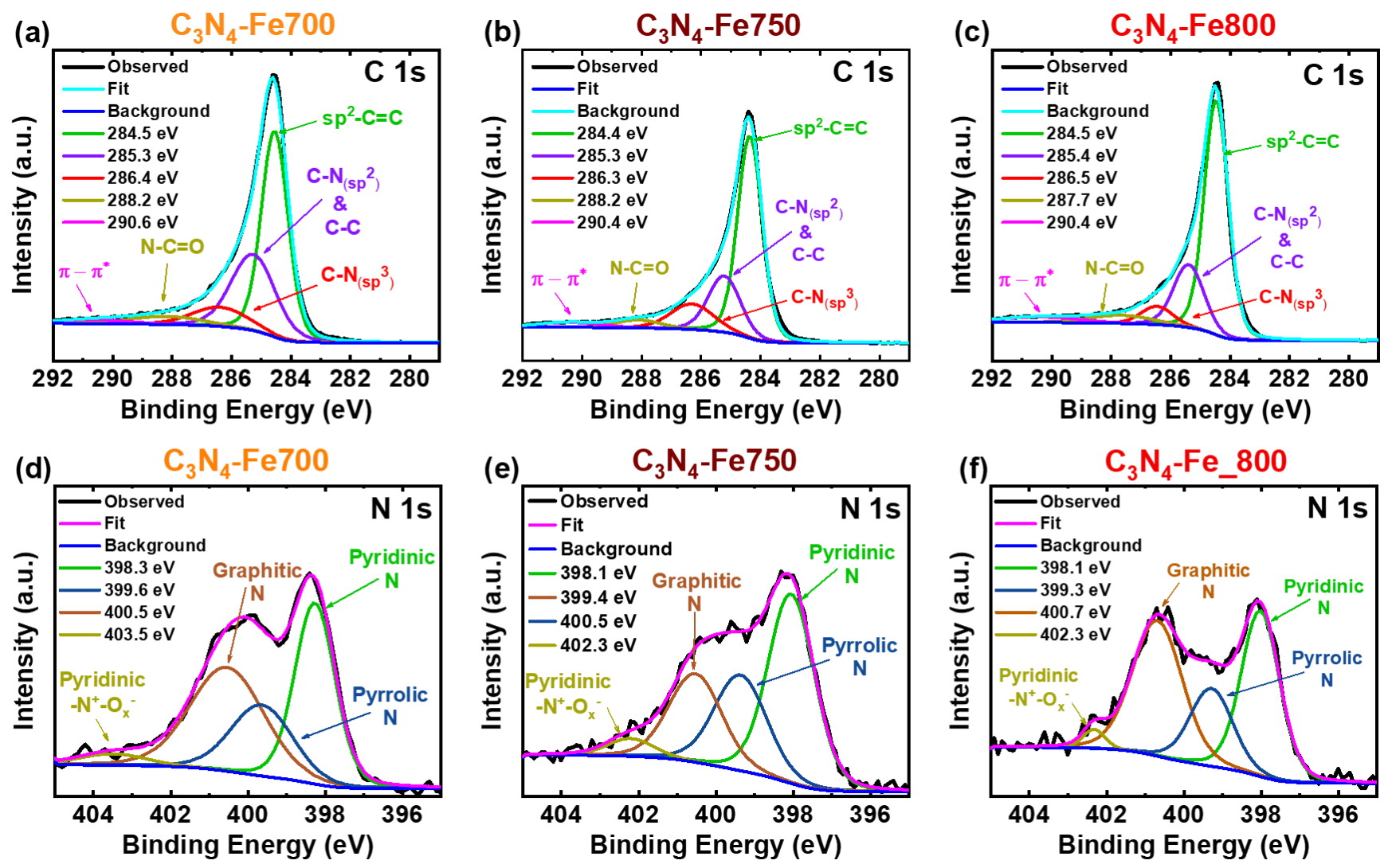

Figure S8. High resolution XPS C 1s (a-c, top row) and N 1s (d-f, bottom row) spectra of $\mathrm{C}_{3} \mathrm{~N}_{4}-\mathrm{Fe} 700$ (a, d), $\mathrm{C}_{3} \mathrm{~N}_{4}-\mathrm{Fe} 750(\mathrm{~b}, \mathrm{e})$, and $\mathrm{C}_{3} \mathrm{~N}_{4}-\mathrm{Fe} 800(\mathrm{c}, \mathrm{f})$. 
Residual Gas Analysis (RGA) of $\mathrm{C}_{3} \mathrm{~N}_{4}-\mathrm{Cu}$ and $\mathrm{C}_{3} \mathrm{~N}_{4}-\mathrm{Fe}$
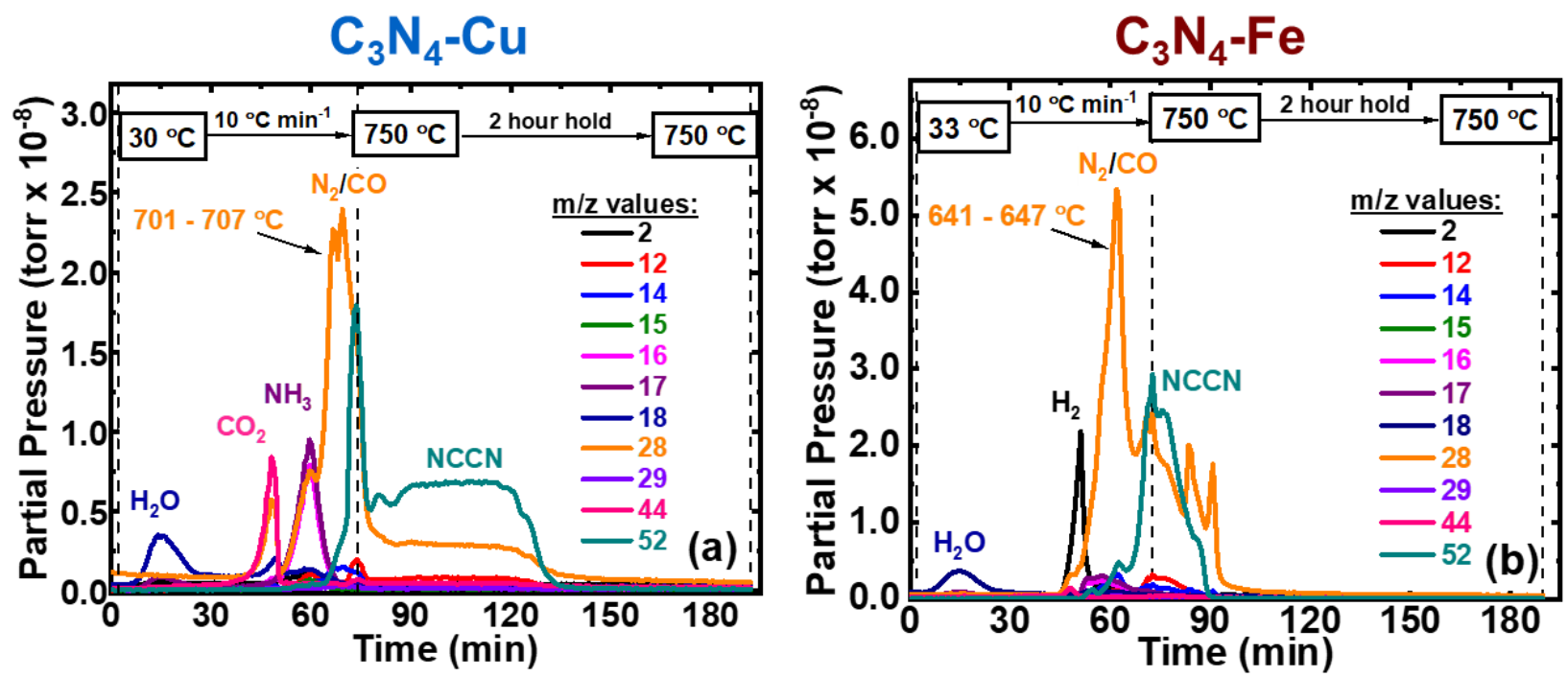

Figure S9. Residual Gas Analysis (RGA) of (a) $\mathrm{C}_{3} \mathrm{~N}_{4}-\mathrm{Cu}$ and (b) $\mathrm{C}_{3} \mathrm{~N}_{4}-\mathrm{Fe}$ under flowing Ar. 
Thermogravimetric Analysis (TGA) curves of $\mathrm{C}_{3} \mathrm{~N}_{4}-\mathrm{Cu} 750$ and $\mathrm{C}_{3} \mathrm{~N}_{4}-\mathrm{Fe} 750$

\section{TGA Parameters:}

The $\mathrm{C}_{3} \mathrm{~N}_{4}-\mathrm{Cu} 750$ and $\mathrm{C}_{3} \mathrm{~N}_{4}-\mathrm{Fe} 750$ carbons $(5.5 \mathrm{mg}$ ) were loaded into $70 \mu \mathrm{L}$ alumina crucibles and ramped to $800{ }^{\circ} \mathrm{C}$ at $10^{\circ} \mathrm{C} \mathrm{min}^{-1}$ under air atmosphere.

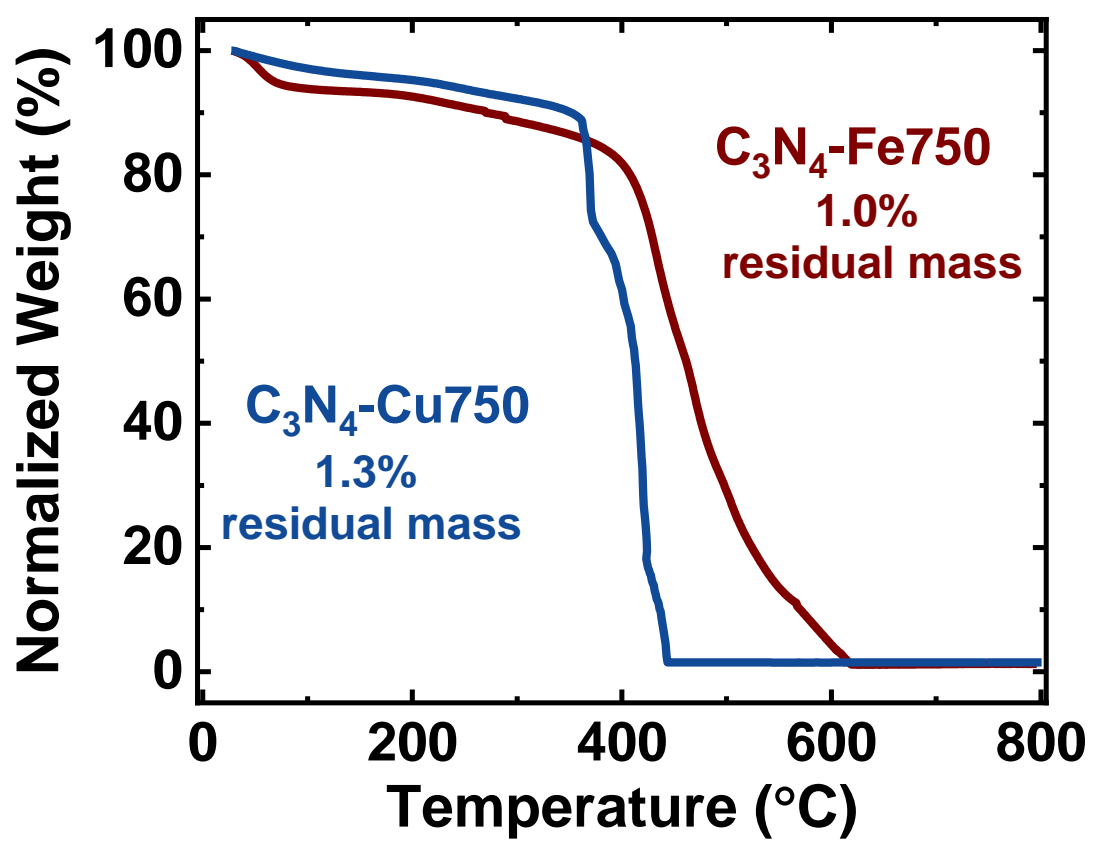

Figure S10. Thermogravimetric analysis (TGA) curves of $\mathrm{C}_{3} \mathrm{~N}_{4}$ $\mathrm{Cu} 750$ (navy curve) and $\mathrm{C}_{3} \mathrm{~N}_{4}-\mathrm{Fe} 750$ (maroon curve). 
Cyclic voltammetry (CV) analysis of bulk $\mathrm{C}_{3} \mathrm{~N}_{4}$
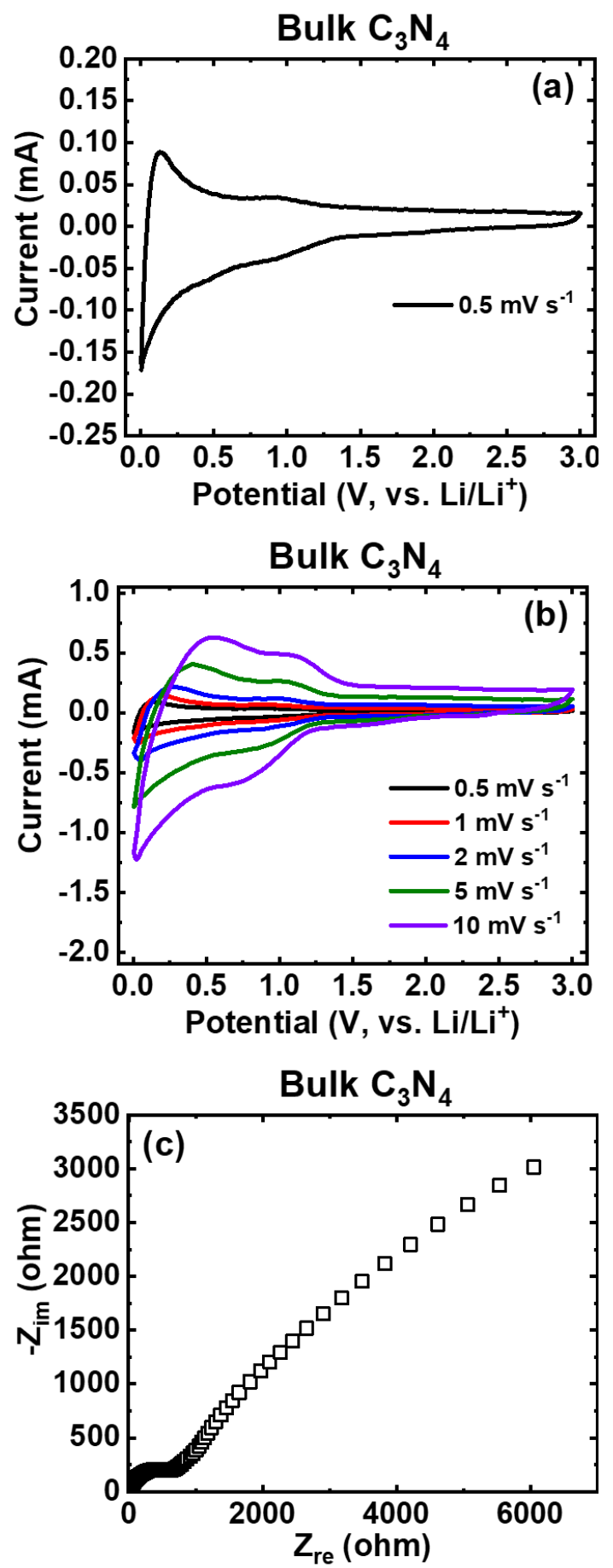

Figure S11. Electrochemical characterization of bulk $\mathrm{C}_{3} \mathrm{~N}_{4}$. (a) $\mathrm{CV}$ curve at $0.5 \mathrm{mV} \mathrm{s}^{-1}$ and (b) $\mathrm{CV}$ curves at varying scan rate from $0.5-10 \mathrm{mV} \mathrm{s}^{-1}$. 
Galvanostatic charge-discharge analysis of bulk $\mathrm{C}_{3} \mathrm{~N}_{4}$
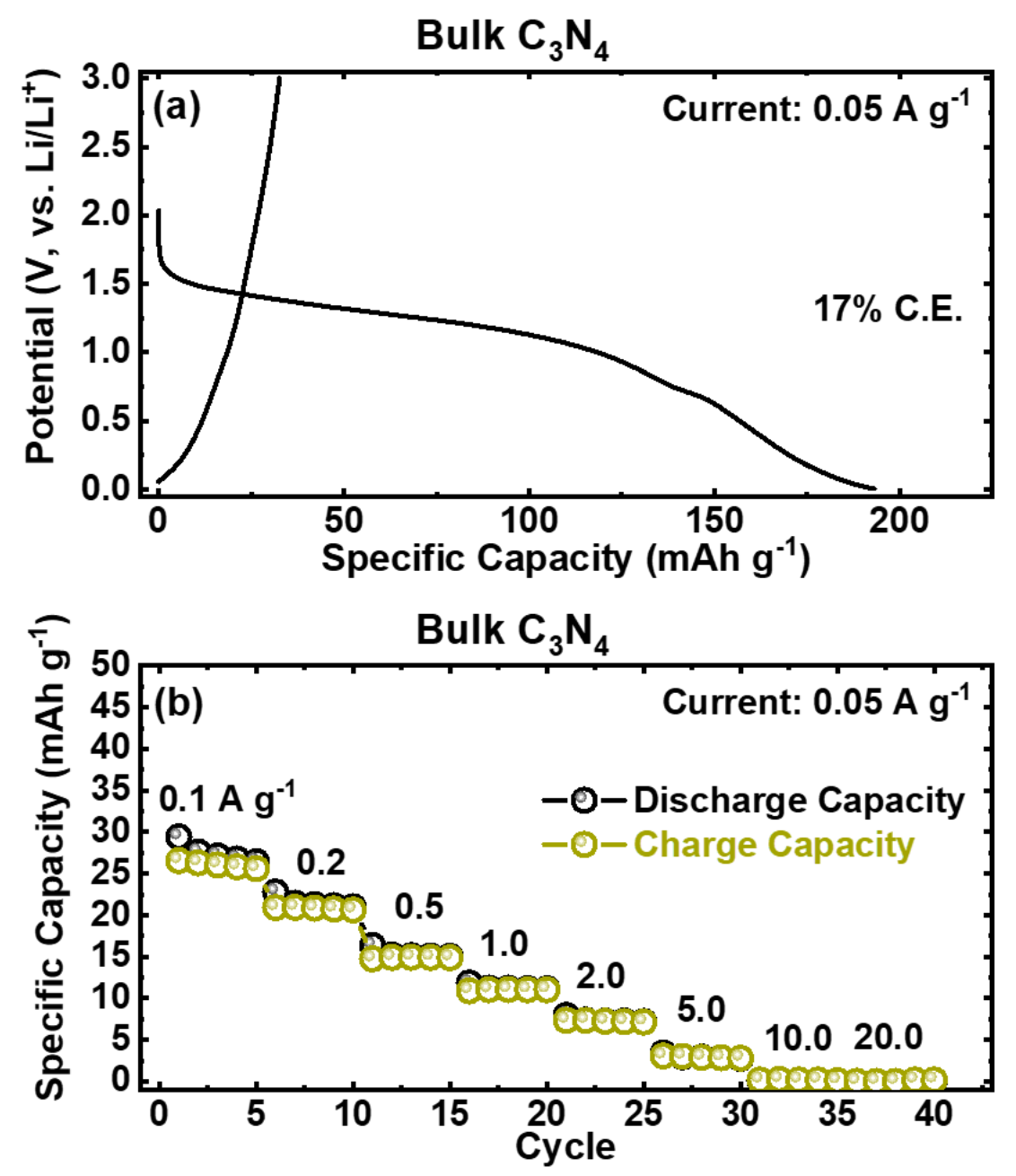

Figure S12. Galvanostatic charge-discharge tests of bulk $\mathrm{C}_{3} \mathrm{~N}_{4}$ showing (a) the first cycle at $0.05 \mathrm{~A} \mathrm{~g}^{-1}$ and (b) rate capability tests cycled at $0.1-20 \mathrm{~A} \mathrm{~g}^{-1}$ in the potential range of $0.005-3.0 \mathrm{~V}$ vs. $\mathrm{Li}^{2} \mathrm{Li}^{+}$. 
Coulombic efficiency vs. cycle for $0.1-20 \mathrm{~A} \mathrm{~g}^{-1}$ (see Figure 7C) and charge-discharge profiles for $\mathrm{C}_{3} \mathrm{~N}_{4}-\mathrm{Cu} 700-800$ at $0.1,0.5,1.0,5.0$, and $20.0 \mathrm{~A} \mathrm{~g} \mathrm{~g}^{-1}$
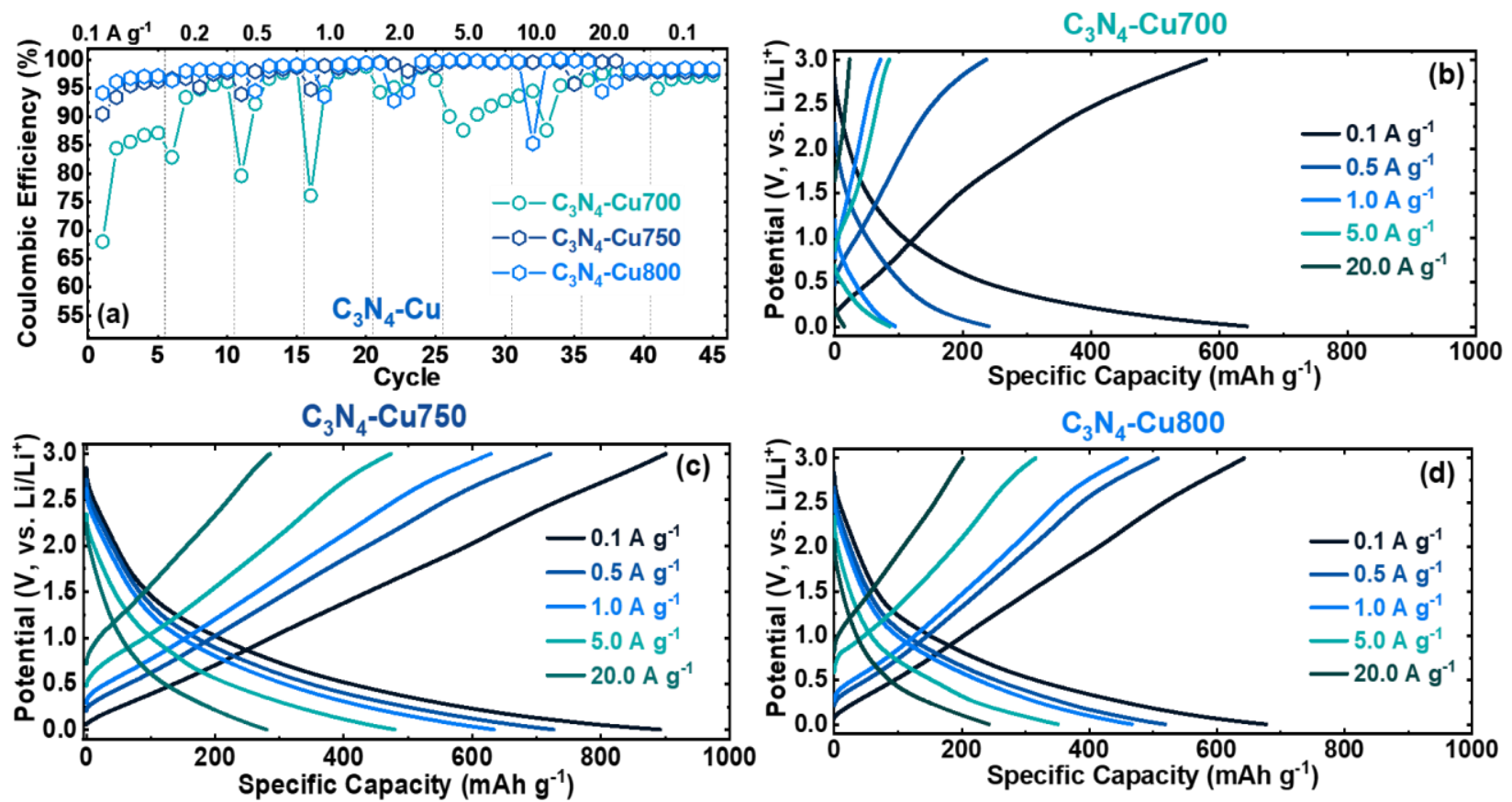

Figure S13. Galvanostatic charge-discharge curves at $0.1,0.5,1.0,5.0$, and $20.0 \mathrm{~A} \mathrm{~g}^{-1}$ for (a) $\mathrm{C}_{3} \mathrm{~N}_{4}-\mathrm{Cu} 700$, (b) $\mathrm{C}_{3} \mathrm{~N}_{4}-\mathrm{Cu} 750$, and (c) $\mathrm{C}_{3} \mathrm{~N}_{4}-\mathrm{Cu} 800$. 
Coulombic efficiency vs. cycle for $0.1-20 \mathrm{~A} \mathrm{~g}^{-1}$ (see Figure 7D) and charge-discharge profiles for $\mathrm{C}_{3} \mathrm{~N}_{4}-\mathrm{Fe} 00-800$ at $0.1,0.5,1.0,5.0$, and $20.0 \mathrm{~A} \mathrm{~g}^{-1}$.
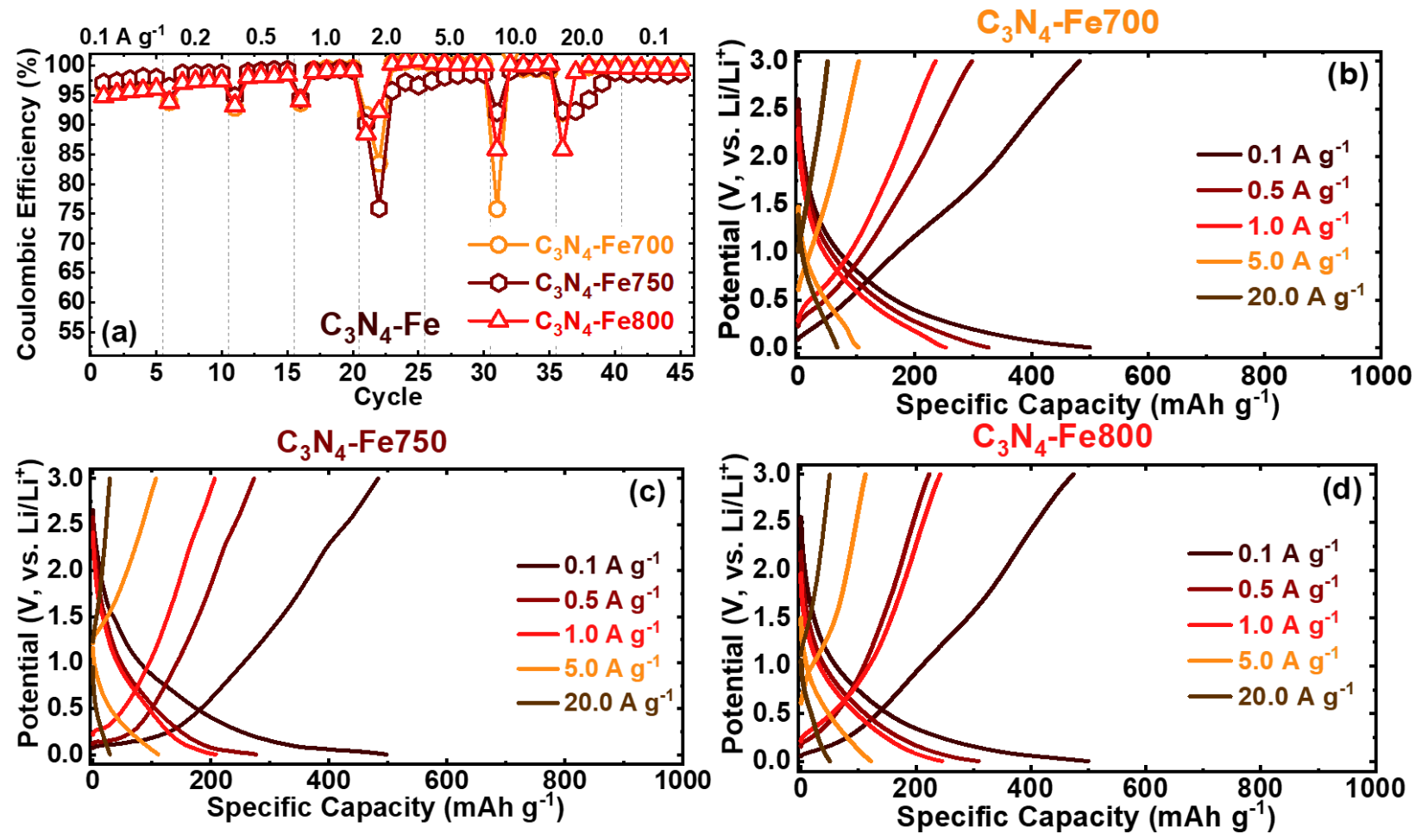

Figure S14. (a) Coulombic efficiency values at $0.1-20 \mathrm{~A} \mathrm{~g}^{-1}$ (see Figure $7 \mathrm{~d}$ ) for $\mathrm{C}_{3} \mathrm{~N}_{4}-\mathrm{Fe} 700-800$. (b-d) Galvanostatic charge-discharge curves at 0.1, 0.5, 1.0, 5.0, and $20.0 \mathrm{~A} \mathrm{~g}^{-1}$ for (b) $\mathrm{C}_{3} \mathrm{~N}_{4}-\mathrm{Fe} 700$, (c) $\mathrm{C}_{3} \mathrm{~N}_{4}$ $\mathrm{Fe} 750$, and (d) $\mathrm{C}_{3} \mathrm{~N}_{4}-\mathrm{Fe} 800$. 

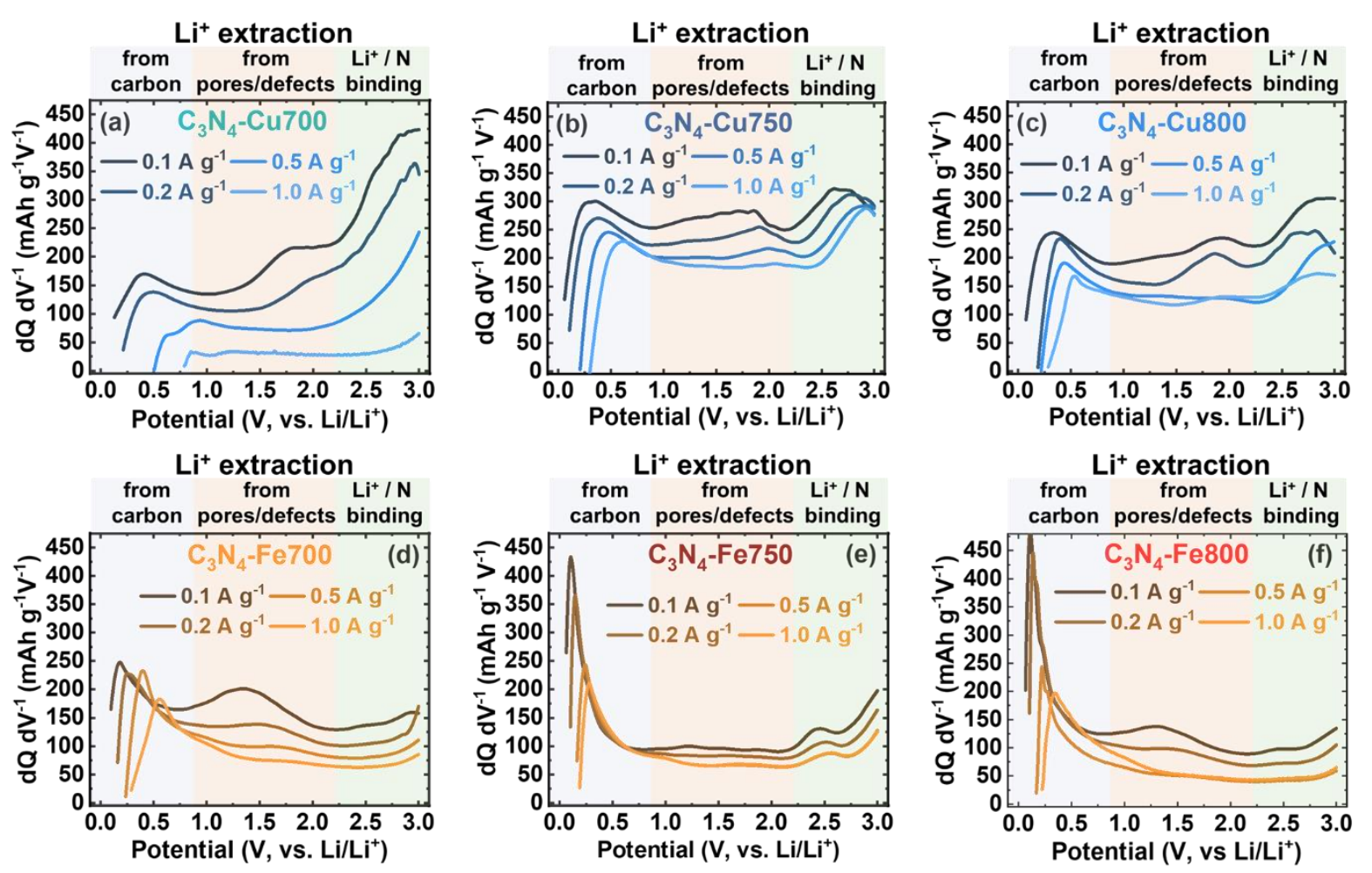

Figure S15. dQ dV ${ }^{-1}$ vs. V plots of the $\mathrm{Li}^{+}$extraction process for (a-c, top row) $\mathrm{C}_{3} \mathrm{~N}_{4}-\mathrm{Cu} 700-800$ and (d-f, bottom row) $\mathrm{C}_{3} \mathrm{~N}_{4}-\mathrm{Fe} 700-800$ at 0.1, 0.2, 0.5, and $1.0 \mathrm{~A} \mathrm{~g}^{-1}$. (a, top left) $\mathrm{C}_{3} \mathrm{~N}_{4}-\mathrm{Cu} 700$, (b, top middle) $\mathrm{C}_{3} \mathrm{~N}_{4}-$ $\mathrm{Cu} 750$, and (c, top right) $\mathrm{C}_{3} \mathrm{~N}_{4}-\mathrm{Cu} 800$. (d, bottom left) $\mathrm{C}_{3} \mathrm{~N}_{4}-\mathrm{Fe} 700$, (e, bottom middle) $\mathrm{C}_{3} \mathrm{~N}_{4}-\mathrm{Fe} 750$, and (f, bottom right) $\mathrm{C}_{3} \mathrm{~N}_{4}-\mathrm{Fe} 800$. 


\section{Cyclic Voltammetry (CV) Analysis of $\mathrm{C}_{3} \mathrm{~N}_{4}-\mathrm{Cu} 700-800$ (Figure S16) and $\mathrm{C}_{3} \mathrm{~N}_{4}-\mathrm{Fe} 700-800$ (Figure S17) carbons}

The charge-storage mechanisms of the $\mathrm{Cu}$ - (Figure $\mathrm{S} 16 \mathrm{a}-\mathrm{c}$ ) and Fe-templated (Figure $\mathrm{S} 17 \mathrm{a}-\mathrm{c})$ carbons were further elucidated by cyclic voltammetry $(\mathrm{CV})$ at scan rate varying of 0.5 $-10 \mathrm{mV} \mathrm{s}^{-1}$ between $0.005-3.0 \mathrm{~V}$ vs. $\mathrm{Li} / \mathrm{Li}^{+}$. For example, we applied a " $b$-value" analysis whereby the measured current, $i$, at a given potential, $\mathrm{V}$, exhibits a power-law relation with the sweep rate, $v$, according to equation $\mathrm{S} 1$ :

$$
i(V)=a v^{b}
$$

With $a$ and $b$ as adjustable parameters. ${ }^{1,2} \mathrm{~A} \log$ - $\log$ plot of current vs. sweep rate for $\mathrm{C}_{3} \mathrm{~N}_{4}$ Cu700-800 (Figure S16d, e) and $\mathrm{C}_{3} \mathrm{~N}_{4}-\mathrm{Fe} 700-800$ (Figure $\mathrm{S} 17 \mathrm{~d}$, e) at a given potential provides insight into the mass-transport character during $\mathrm{Li}^{+}$extraction. In this analysis, a $b$-value of 1.0 corresponds to a diffusion-independent process, such as a capacitive-charge storage or faradic electron transfer between $\mathrm{Li}^{+}$and the electrode surface. On the other hand, a $b$-value of 0.5 represents a process controlled be semi-infinite diffusion, such as $\mathrm{Li}^{+}$intercalation/deintercalation from carbon layers. ${ }^{1,2}$

From the same analysis, the relation between the $\mathrm{Li}^{+}$diffusion coefficient can be estimated using the Randles-Sevcik Equation (Equation S2): ${ }^{3-5}$

$$
I_{p}=.4463 n^{3 / 2} F^{3 / 2} C_{L i}+S R^{-1} T^{-1}\left(D_{L i^{+}}\right)^{\frac{1}{2}}(v)^{1 / 2}
$$

Where $I_{p}$ is the peak current at a given voltage, $n$ is the charge-transfer number, $\mathrm{F}$ is Faraday's constant, $\mathrm{C}_{\mathrm{Li}}{ }^{+}$is the Li-ion concentration, $\mathrm{S}$ is the surface area per weight of active material, $\mathrm{R}$ is the gas constant, $\mathrm{T}$ is the temperature, $\mathrm{DLi}^{+}$is the Li-ion diffusion coefficient, and $v$ is the scan rate. Calculated $\mathrm{D}_{\mathrm{Li}}{ }^{+}$at different potentials during $\mathrm{Li}^{+}$extraction for $\mathrm{C}_{3} \mathrm{~N}_{4}-\mathrm{Cu} 700-800$ and $\mathrm{C}_{3} \mathrm{~N}_{4}{ }^{-}$ Fe700-800 are shown in Figure S16f and Figure S17f, respectively. 

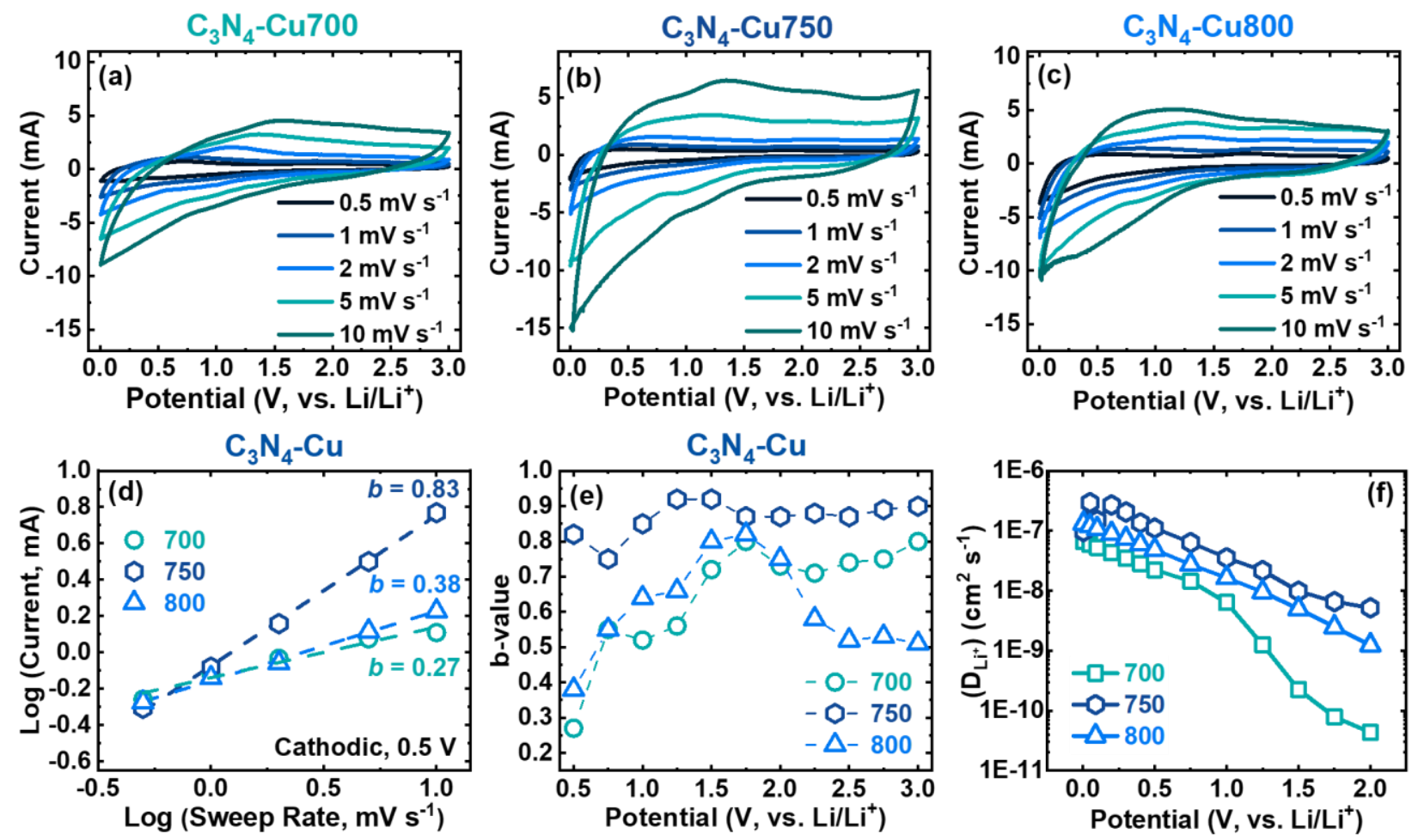

Figure S16. Cyclic voltammetry (CV) curves at $0.5,1,2,5$, and $10 \mathrm{mV} \mathrm{s}^{-1}$ for (a, top left) $\mathrm{C}_{3} \mathrm{~N}_{4}-\mathrm{Cu} 700$, (b, top middle) $\mathrm{C}_{3} \mathrm{~N}_{4}-\mathrm{Cu} 750$, and (c, top right) $\mathrm{C}_{3} \mathrm{~N}_{4}-\mathrm{Cu} 800$. (d) Log-log plots of current vs. sweep rate at 0.5 $\mathrm{V}$ for $\mathrm{C}_{3} \mathrm{~N}_{4}-\mathrm{Cu} 700-800$. (e) B-value analysis at different potentials vs. $\mathrm{Li} / \mathrm{Li}^{+}$for $\mathrm{C}_{3} \mathrm{~N}_{4}-\mathrm{Cu} 700-800$ using equation S1. (f) Lithium diffusion coefficient at different potentials vs. $\mathrm{Li}^{2} \mathrm{Li}^{+}$for $\mathrm{C}_{3} \mathrm{~N}_{4}-\mathrm{Cu} 700-800$ calculated from equation $\mathrm{S} 2$. 

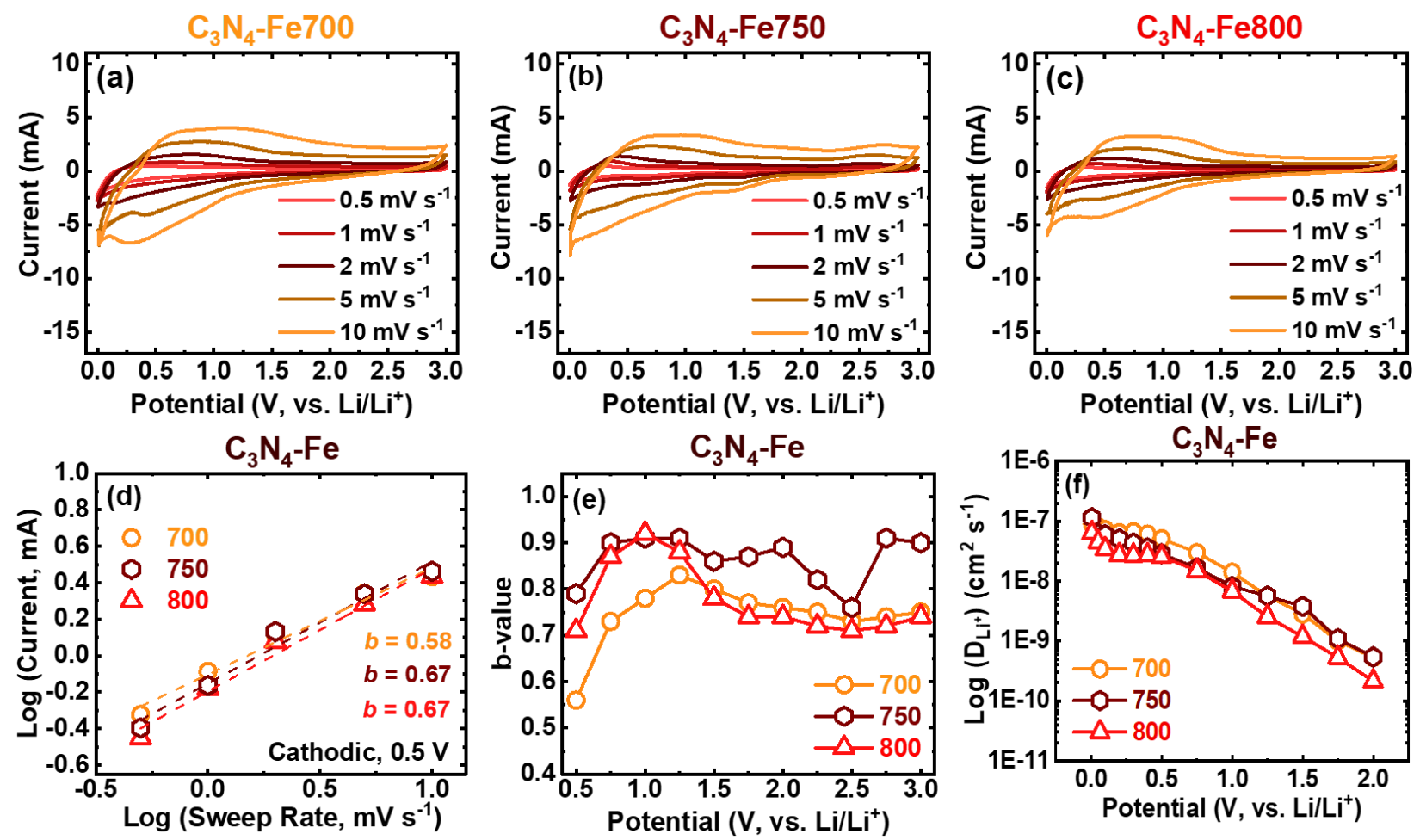

Figure S17. Cyclic voltammetry (CV) curves at $0.5,1,2,5$, and $10 \mathrm{mV} \mathrm{s}^{-1}$ for (a, top left) $\mathrm{C}_{3} \mathrm{~N}_{4}-\mathrm{Fe} 700$, (b, top middle) $\mathrm{C}_{3} \mathrm{~N}_{4}-\mathrm{Fe} 750$, and (c, top right) $\mathrm{C}_{3} \mathrm{~N}_{4}-\mathrm{Fe} 800$. (d) Log-log plots of current vs. sweep rate at 0.5 $\mathrm{V}$ for $\mathrm{C}_{3} \mathrm{~N}_{4}-\mathrm{Fe} 700-800$. (e) B-value analysis at different potentials vs. $\mathrm{Li} / \mathrm{Li}^{+}$for $\mathrm{C}_{3} \mathrm{~N}_{4}-\mathrm{Fe} 700-800$ using equation S1. (f) Lithium diffusion coefficient at different potentials vs. $\mathrm{Li}^{2} \mathrm{Li}^{+}$for $\mathrm{C}_{3} \mathrm{~N}_{4}-\mathrm{Fe} 700-800$ calculated from equation $\mathrm{S} 2$. 
Charge-discharge curves and coulombic efficiency plots for $\mathrm{C}_{3} \mathrm{~N}_{4}-\mathrm{Cu}$ carbons for 200 cycles at $0.1 \mathrm{~A} \mathrm{~g}^{-1}\left(0.2 \mathrm{~mA} \mathrm{~cm}^{-2}\right)$
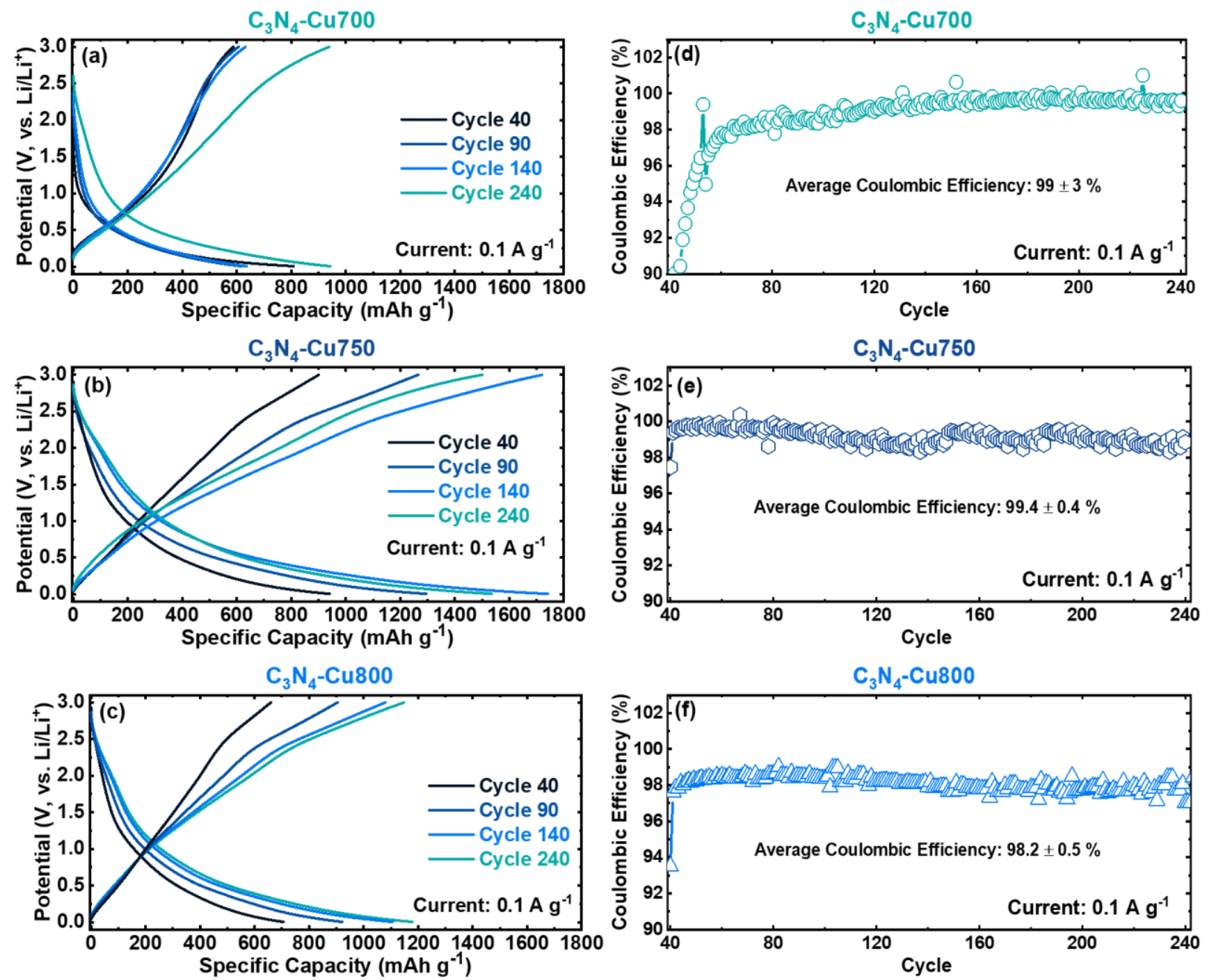

Figure S18. Galvanostatic charge-discharge tests (a-c, left column) and coulombic efficiency plots (d-f, right column) over 200 cycles at $0.1 \mathrm{~A} \mathrm{~g}^{-1}$ for $(\mathrm{a}, \mathrm{d}) \mathrm{C}_{3} \mathrm{~N}_{4}-\mathrm{Cu} 700$, (b, e) $\mathrm{C}_{3} \mathrm{~N}_{4}-\mathrm{Cu} 750$, and (c, f) $\mathrm{C}_{3} \mathrm{~N}_{4}-$ $\mathrm{Cu} 800$. 

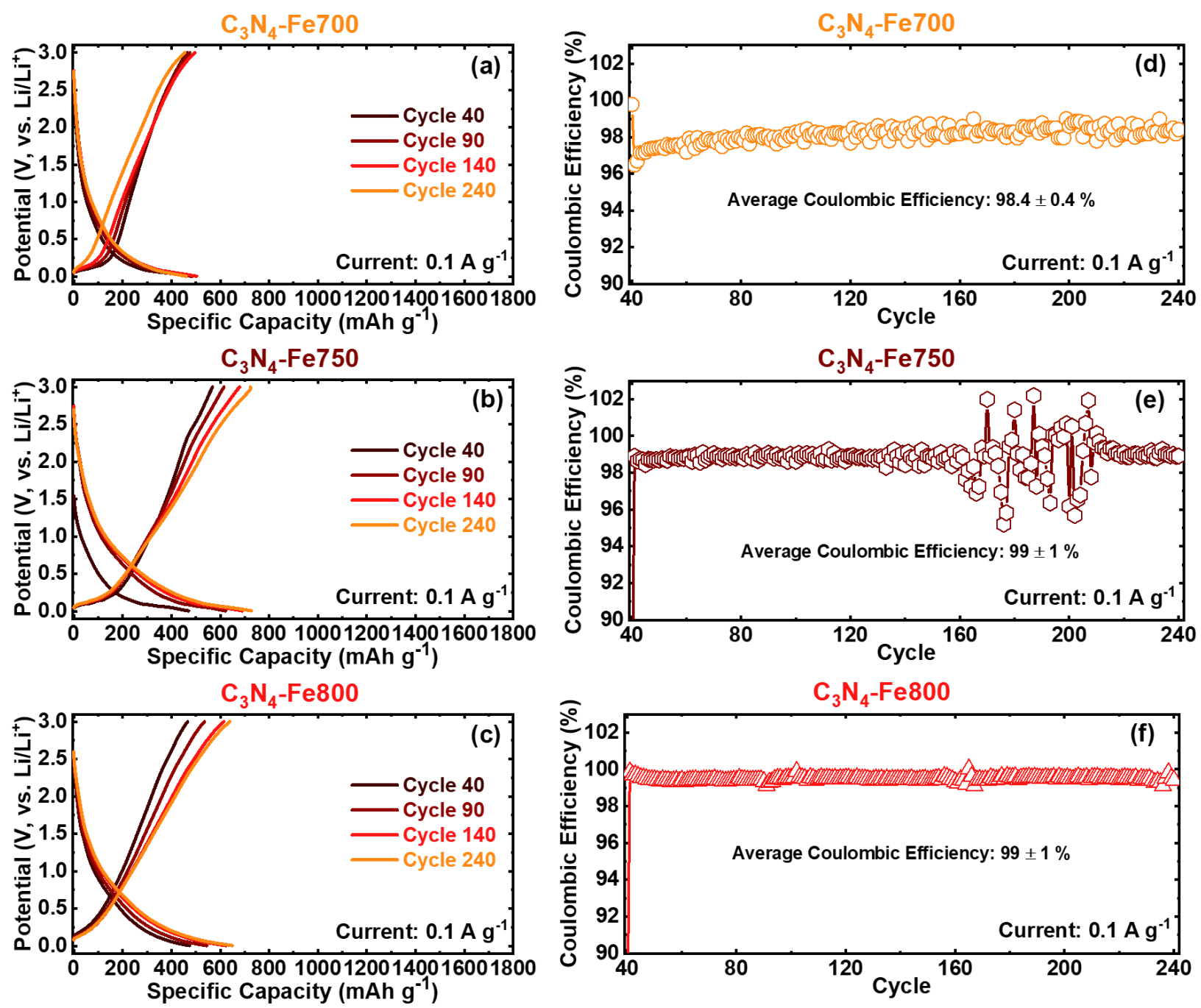

Figure S19. Galvanostatic charge-discharge tests (a-c, left column) and coulombic efficiency plots (d-f, right column) over 200 cycles at $0.1 \mathrm{~A} \mathrm{~g} \mathrm{~g}^{-1}$ for (a, d) $\mathrm{C}_{3} \mathrm{~N}_{4}-\mathrm{Fe} 700$, (b, e) $\mathrm{C}_{3} \mathrm{~N}_{4}-\mathrm{Fe} 750$, and (c, f) $\mathrm{C}_{3} \mathrm{~N}_{4}-\mathrm{Fe} 800$. 

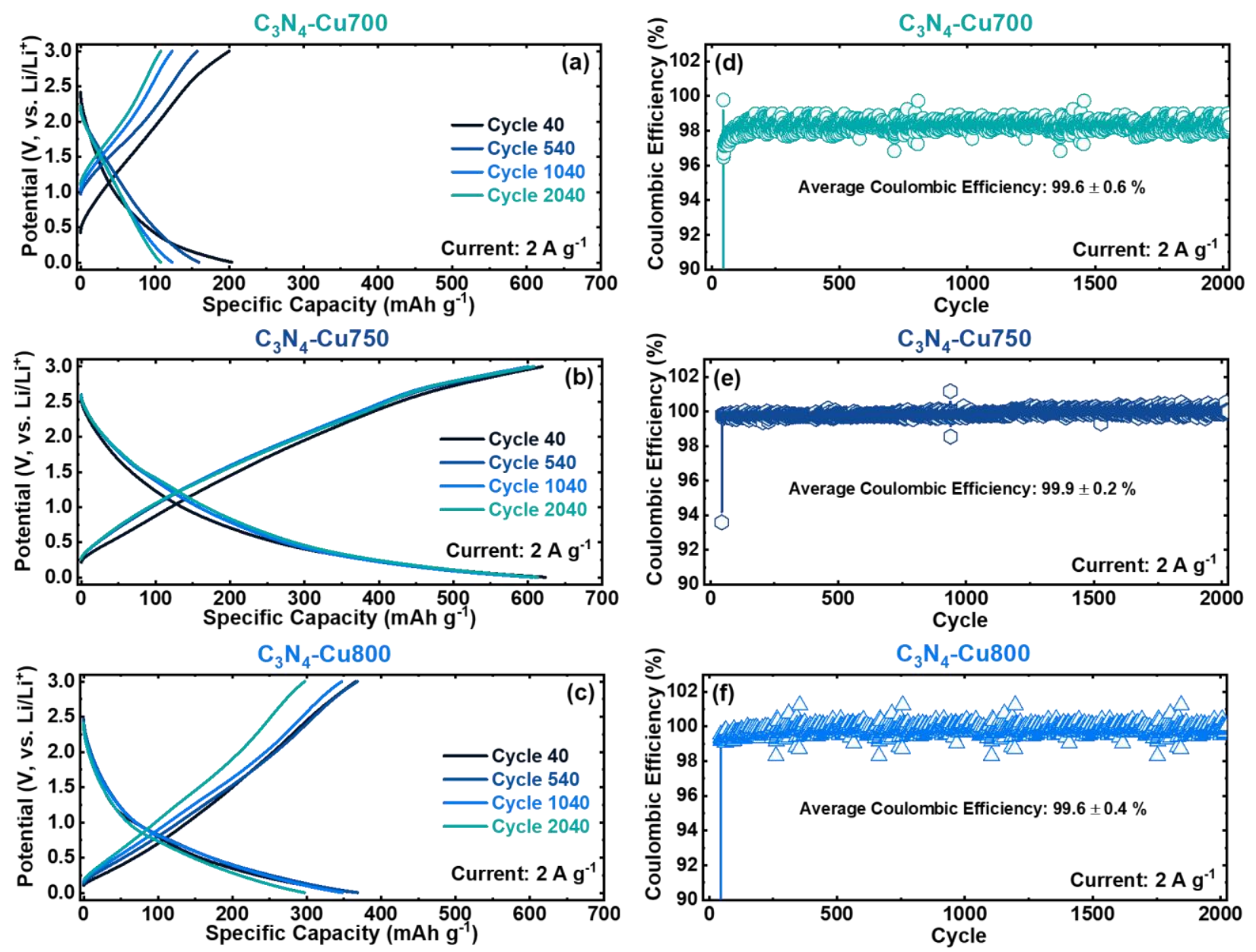

Figure S20. Galvanostatic charge-discharge tests (a-c, left column) and coulombic efficiency plots (d-f, right column) at over 2000 cycles at $2.0 \mathrm{~A} \mathrm{~g}^{-1}\left(4.0 \mathrm{~mA} \mathrm{~cm}^{-2}\right)$ for (a, d) $\mathrm{C}_{3} \mathrm{~N}_{4}-\mathrm{Cu} 700$, (b, e) $\mathrm{C}_{3} \mathrm{~N}_{4}-\mathrm{Cu} 750$, and (c, f) $\mathrm{C}_{3} \mathrm{~N}_{4}-\mathrm{Cu} 800$. 

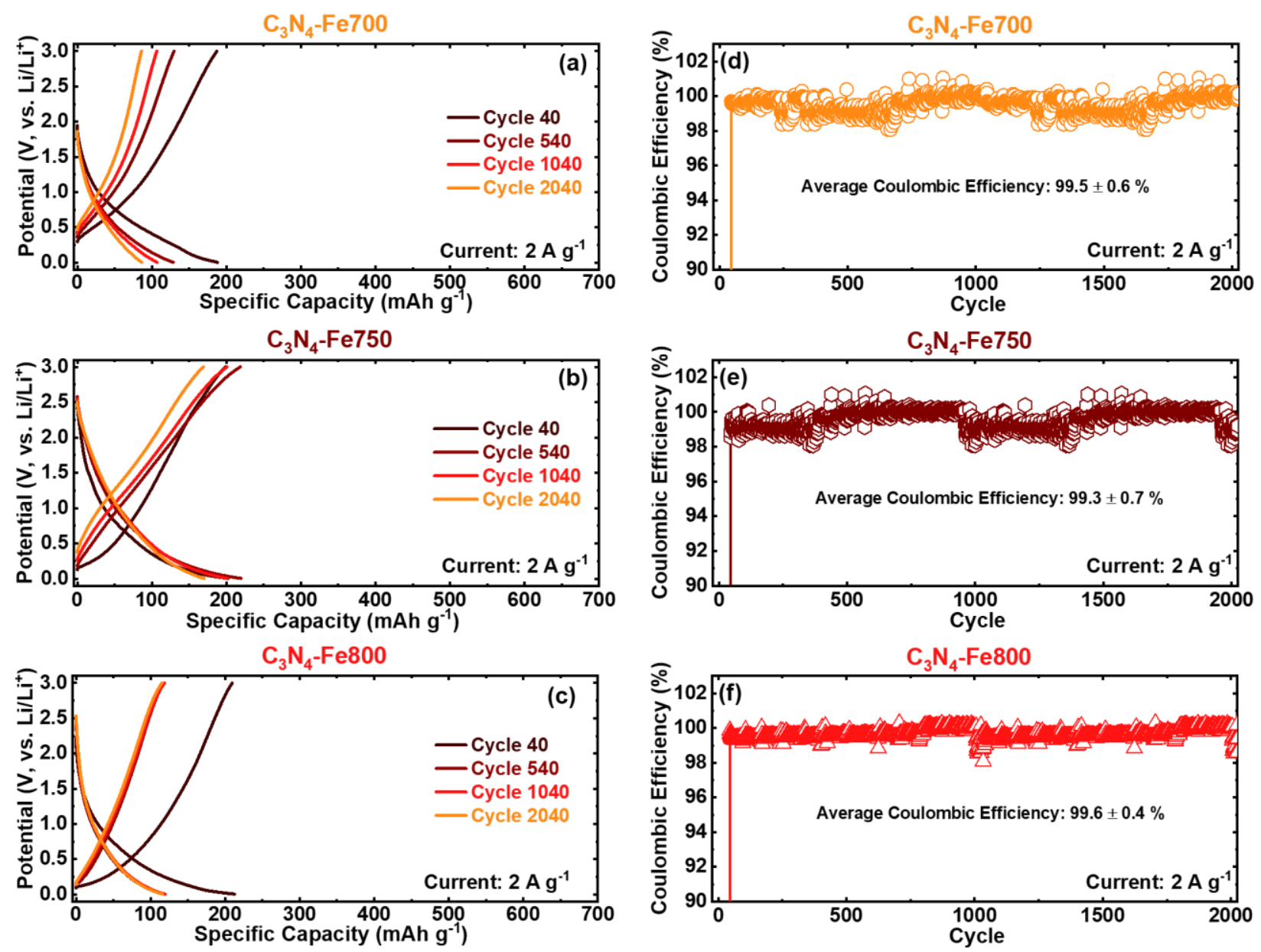

Figure S21. Galvanostatic charge-discharge profiles (a-c, left column) and coulombic efficiency plots (df, right column) over 2000 cycles at $2.0 \mathrm{~A} \mathrm{~g}^{-1}\left(4.0 \mathrm{~mA} \mathrm{~cm}^{-2}\right)$ for (a, d) $\mathrm{C}_{3} \mathrm{~N}_{4}-\mathrm{Fe} 700$, (b, e) $\mathrm{C}_{3} \mathrm{~N}_{4}-\mathrm{Fe} 750$, and (c, f) $\mathrm{C}_{3} \mathrm{~N}_{4}-\mathrm{Fe} 800$. 
Specific Capacity vs. Cycle between 0.1 to $20 \mathrm{~A} \mathrm{~g}^{-1}$ for $\mathrm{C}_{3} \mathrm{~N}_{4}-\mathrm{Cu} 750$ at different mass loading

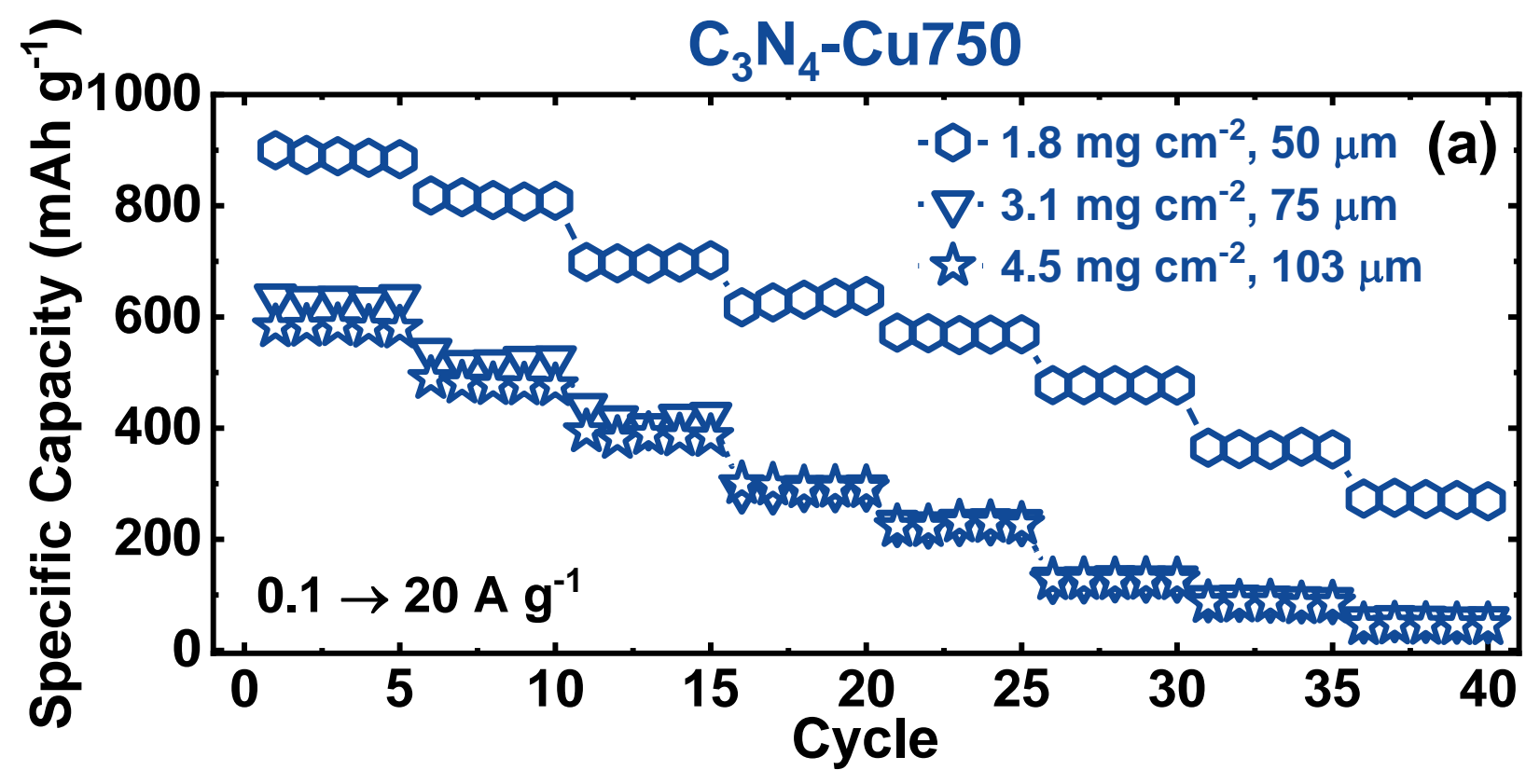

Figure S22. (a) Rate Capability tests between $0.1-20 \mathrm{~A} \mathrm{~g}^{-1}$ for C3N4-Cu750 at $1.8 \mathrm{mg} \mathrm{cm}^{-2}$ (navy hexagons), $3.1 \mathrm{mg} \mathrm{cm}^{-2}$ (navy downward triangles), and $4.5 \mathrm{mg} \mathrm{cm}^{-2}$ (navy stars). 
Table S3. Parameters compiled from previous works used to construct Figure 10b.

\begin{tabular}{|c|c|c|c|c|c|c|c|}
\hline Material & $\begin{array}{l}\text { Heteroatom } \\
\text { content } \\
(\%)\end{array}$ & $\begin{array}{l}\text { Reversible } \\
\text { Capacity } \\
\left(\mathrm{mAh} \mathrm{g}^{-1}\right)\end{array}$ & $\begin{array}{l}\text { Specific } \\
\text { Current } \\
\left(\mathrm{A} \mathrm{g}^{-1}\right)\end{array}$ & $\begin{array}{c}\text { Mass } \\
\text { loading } \\
\left(\mathrm{mg} \mathrm{cm}^{-2}\right)\end{array}$ & 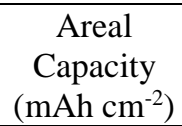 & $\begin{array}{c}\text { Current } \\
\text { Density } \\
\left(\mathrm{mA} \mathrm{cm}^{-2}\right)\end{array}$ & Reference \\
\hline $\begin{array}{l}\text { Melamine/ } \\
\text { Phytic Acid } \\
\text { co-doped } \\
\text { graphene } \\
\end{array}$ & $\begin{array}{c}15 \% \mathrm{~N} \\
9 \% \mathrm{P}\end{array}$ & $\begin{array}{l}946 \\
595\end{array}$ & $\begin{array}{c}0.5 \\
10.0\end{array}$ & $\sim 1$ & $\begin{array}{l}0.95 \\
0.60\end{array}$ & $\begin{array}{c}0.5 \\
20.0\end{array}$ & 20 \\
\hline $\begin{array}{l}\text { Pyrolyzed } \\
\text { melamine } \\
\text { foam }\end{array}$ & $5 \% \mathrm{~N}$ & $\begin{array}{l}416 \\
240\end{array}$ & $\begin{array}{l}0.1 \\
2.0\end{array}$ & 3.2 & $\begin{array}{l}1.33 \\
0.77\end{array}$ & $\begin{array}{c}0.32 \\
6.4\end{array}$ & 24 \\
\hline $\begin{array}{c}\text { Pyrolyzed } \\
\text { zeolitic } \\
\text { imidazolate } \\
\text { MOF } \\
\end{array}$ & $18 \% \mathrm{~N}$ & $\begin{array}{l}2163 \\
1182\end{array}$ & $\begin{array}{l}0.1 \\
1.6\end{array}$ & $<1$ & $\begin{array}{l}2.16 \\
1.18\end{array}$ & $\begin{array}{l}0.1 \\
1.6\end{array}$ & 40 \\
\hline $\begin{array}{c}\text { Wheat-stalk } \\
\text { derived } \\
\text { Graphite } \\
\text { Nanosheets } \\
\end{array}$ & $1.5 \% \mathrm{O}$ & $\begin{array}{l}502 \\
305\end{array}$ & $\begin{array}{l}0.04 \\
0.74\end{array}$ & $\sim 1$ & $\begin{array}{l}0.50 \\
0.31\end{array}$ & $\begin{array}{l}0.04 \\
0.74\end{array}$ & 41 \\
\hline $\begin{array}{c}\text { Pyrolyzed } \\
\text { glucose-urea } \\
\text { templated N- } \\
\text { doped carbon }\end{array}$ & $7 \% \mathrm{~N}$ & $\begin{array}{c}1406 \\
344\end{array}$ & $\begin{array}{c}0.1 \\
10.0\end{array}$ & 0.54 & $\begin{array}{l}0.76 \\
0.19\end{array}$ & $\begin{array}{c}0.05 \\
1.3\end{array}$ & 42 \\
\hline $\begin{array}{c}\text { Ni- } \\
\text { Nanoparticle } \\
\text { embedded } \\
\text { Carbon } \\
\text { Nanosheets } \\
\end{array}$ & $20 \% \mathrm{Ni}$ & $\begin{array}{l}775 \\
400\end{array}$ & $\begin{array}{l}0.1 \\
1.0\end{array}$ & ca. 1 & $\begin{array}{l}0.78 \\
0.40\end{array}$ & $\begin{array}{l}0.1 \\
1.0\end{array}$ & 43 \\
\hline $\begin{array}{l}\text { Zinc-templated } \\
\text { pyrolyzed } \\
\text { glucose carbon }\end{array}$ & $16 \% \mathrm{O}$ & $\begin{array}{c}1041 \\
370\end{array}$ & $\begin{array}{c}0.1 \\
10.0\end{array}$ & 0.53 & $\begin{array}{l}0.56 \\
0.20\end{array}$ & $\begin{array}{c}0.05 \\
5.3\end{array}$ & 44 \\
\hline $\begin{array}{c}\text { MgO- } \\
\text { templated } \\
\text { Carbon } \\
\text { Nanocages }\end{array}$ & Not reported & $\begin{array}{r}900 \\
190\end{array}$ & $\begin{array}{c}0.1 \\
10\end{array}$ & $0.25-0.5$ & $\begin{array}{l}0.51 \\
0.09\end{array}$ & $\begin{array}{c}0.05 \\
5\end{array}$ & 45 \\
\hline $\begin{array}{c}\text { Coconut-oil } \\
\text { derived } \\
\text { Carbon } \\
\text { Nanoparticles } \\
\end{array}$ & $17 \% \mathrm{O}$ & $\begin{array}{l}742 \\
295\end{array}$ & $\begin{array}{l}0.1 \\
1.0\end{array}$ & $\sim 1.5$ & $\begin{array}{l}1.11 \\
0.44\end{array}$ & $\begin{array}{c}0.15 \\
1.5\end{array}$ & 46 \\
\hline $\begin{array}{l}\text { Ni/Porphyra } \\
\text { derived Porous } \\
\text { Carbon }\end{array}$ & $5.3 \% \mathrm{~N}$ & $\begin{array}{c}1006 \\
278\end{array}$ & $\begin{array}{l}0.1 \\
5.0\end{array}$ & 1.3 & $\begin{array}{l}1.31 \\
0.36\end{array}$ & $\begin{array}{c}0.13 \\
6.5\end{array}$ & 47 \\
\hline $\begin{array}{c}\text { Protein- } \\
\text { derived } \\
\text { Mesoporous } \\
\text { Carbon } \\
\end{array}$ & $10.1 \% \mathrm{~N}$ & $\begin{array}{c}1773 \\
204\end{array}$ & $\begin{array}{l}0.1 \\
4.0\end{array}$ & 0.51 & $\begin{array}{l}0.90 \\
0.10\end{array}$ & $\begin{array}{c}0.05 \\
2.0\end{array}$ & 48 \\
\hline $\mathrm{C}_{3} \mathrm{~N}_{4}-\mathrm{Cu} 750$ & $30 \% \mathrm{~N}$ & $\begin{array}{l}900 \\
273\end{array}$ & $\begin{array}{l}0.1 \\
20\end{array}$ & 1.8 & $\begin{array}{l}1.6 \\
0.5\end{array}$ & $\begin{array}{l}0.2 \\
36\end{array}$ & $\begin{array}{l}\text { This } \\
\text { Work }\end{array}$ \\
\hline $\mathrm{C}_{3} \mathrm{~N}_{4}-\mathrm{Cu} 750$ & $30 \% \mathrm{~N}$ & $\begin{array}{l}589 \\
135\end{array}$ & $\begin{array}{l}0.1 \\
20\end{array}$ & 4.5 & $\begin{array}{l}2.8 \\
0.2\end{array}$ & $\begin{array}{c}0.45 \\
90\end{array}$ & $\begin{array}{l}\text { This } \\
\text { Work }\end{array}$ \\
\hline
\end{tabular}


Electrochemical Impedance Spectroscopy (EIS) Analysis of bulk $\mathrm{C}_{3} \mathrm{~N}_{4}, \mathrm{C}_{3} \mathrm{~N}_{4}-\mathrm{Cu}$, and $\mathrm{C}_{3} \mathrm{~N}_{4-}$ Fe carbons
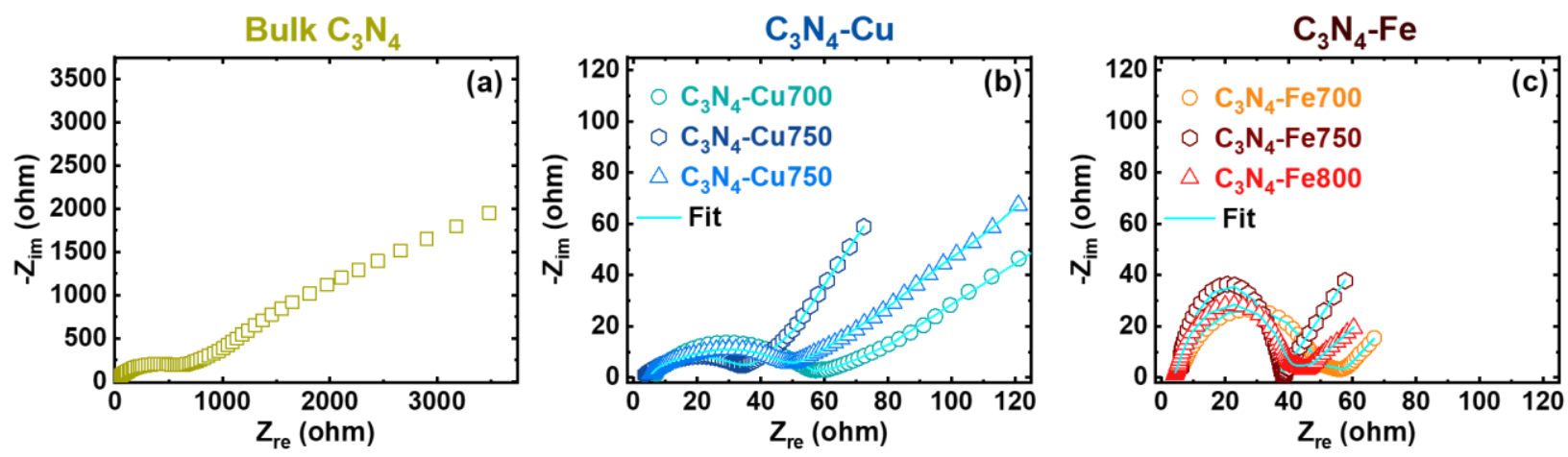

(d)

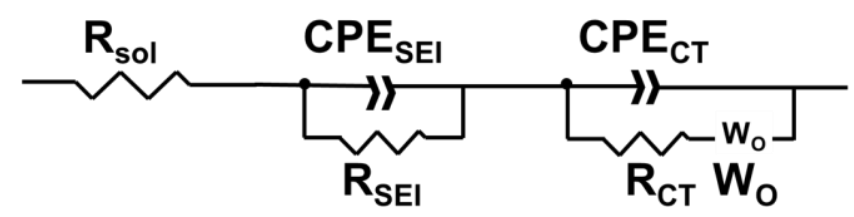

Figure S23. Electrochemical impedance spectroscopy (EIS) Nyquist plots for (a) bulk $\mathrm{C}_{3} \mathrm{~N}_{4}$, (b) $\mathrm{C}_{3} \mathrm{~N}_{4}$ Cu700-800, and (c) $\mathrm{C}_{3} \mathrm{~N}_{4}-\mathrm{Fe} 700-800$. (d) The equivalent circuit used to fit the observed EIS spectra. 


\section{References}

(1) Ko, J. S.; Sassin, M. B.; Parker, J. F.; Rolison, D. R.; Long, J. W. Combining Battery-Like and Pseudocapacitive Charge Storage in 3D $\mathrm{MnO}_{\mathrm{x}} @$ Carbon Electrode Architectures for Zinc-Ion Cells. Sustain. Energy Fuels 2017, 2, 626-636.

(2) Ma, C.; Deng, C.; Liao, X.; He, Y.; Ma, Z.; Xiong, H. Nitrogen and Phosphorus Codoped Porous Carbon Framework as Anode Material for High Rate Lithium-Ion Batteries. ACS Appl. Mater. Interfaces 2018, 10, 36969-36975.

(3) Lee, Y. S.; Ryu, K. S. Study of the Lithium Diffusion Properties and High Rate Performance of $\mathrm{TiNb}_{6} \mathrm{O}_{17}$ as an Anode in Lithium Secondary Battery. Sci. Rep. 2017, 7, $1-13$.

(4) Tang, S. B.; Lai, M. O.; Lu, L. Li-Ion Diffusion in Highly (003) Oriented $\mathrm{LiCoO}_{2}$ Thin Film Cathode Prepared by Pulsed Laser Deposition. J. Alloys Compd. 2008, 449, 300303.

(5) Park, C. K.; Park, S. Bin; Oh, S. H.; Jang, H.; Cho, W. Li Ion Diffusivity and Improved Electrochemical Performances of the Carbon Coated $\mathrm{LiFePO}_{4}$. Bull. Korean Chem. Soc. 2011, 32, 836-840. 